\title{
El trabajo doméstico y de cuidados en las parejas de doble ingreso. Análisis comparativo entre España, Argentina y Chile
}

\author{
Màrius Domínguez Amorós \\ Universitat de Barcelona. Departament de Sociologia \\ mariusdominguez@ub.edu \\ ORCID: 0000-0003-2225-4987

\section{Leticia Muñiz} \\ CONICET. CIMeCS-IdIHCS / Universidad Nacional de la Plata \\ lmunizterra@conicet.gov.ar \\ ORCID: 0000-0002-1812-4496
}

Gabriela Rubilar Donoso

Universidad de Chile

grubilar@uchile.cl

ORCID: 0000-0002-4635-9380

Recepción: 17-04-2018

Aceptación: 11-10-2018

Publicación: 14-12-2018

\section{Resumen}

Este artículo analiza comparativamente las brechas de género en el trabajo no remunerado realizado en los hogares en Argentina, Chile y España. Concretamente se analiza la distribución del trabajo doméstico y de cuidados entre los miembros del hogar a partir del análisis del uso del tiempo, y se profundiza en los factores explicativos de la distribución del trabajo doméstico y de cuidados. Utilizando datos de encuestas nacionales del uso del tiempo (EET-2010 de España; TRN-2013 de Argentina y ENUT-2015 de Chile), se estima un modelo de regresión a una muestra de hogares de parejas de doble ingreso. Además, se complementa con el análisis de los factores macrosociales que inciden en la brecha de género. Los resultados muestran que en los tres países las mujeres realizan el $70 \%$ del trabajo doméstico y de cuidados del hogar. Respecto a los factores explicativos, la debilidad de las variables relacionadas con los recursos relativos inciden en la necesidad de incorporar la perspectiva teórica de los roles de género y los elementos contextuales para dar cuenta de la brecha de género en el interior de los hogares. La relevancia de la disponibilidad de tiempos y el aporte de ingresos de las mujeres señalan la importancia de acciones que procuren una mayor independencia económica de las mujeres y el fortalecimiento de marcos legales que resguarden condiciones salariales igualitarias, ya que constituyen aspectos que inciden en los determinantes de la división del trabajo doméstico y de cuidados revisados en este trabajo.

Palabras clave: trabajo no remunerado del hogar; uso del tiempo; género; análisis comparativo 
Abstract. Domestic and care work in dual-earner couples: Comparative analysis of Spain, Argentina and Chile

This article analyses the unpaid household work gender gap in Spain, Argentina and Chile. Specifically, this study explores the distribution of domestic and care work among household members and examines in depth the relevant factors of women's and men's participation in this work. Employing time use surveys (Spanish EET-2010, Argentinian TRN-2013 and Chilean ENUT-2015), a regression model is estimated for a sample of dual-income couples. Additionally, the study analyses the macrosocial factors that affect the gender gap. The results show that women perform $70 \%$ of domestic and care work and confirm the weakness of the variables related to relative resources, thus suggesting the need to incorporate the gender role perspective and structural, institutional, and cultural factors in explaining gender gaps in unpaid work. The relevance of women's time availability and income contribution points to the importance of actions aimed at promoting the economic independence of women and strengthening legal frameworks that protect equal pay conditions for women. These elements are key to explaining men's and women's decisions on how to distribute their time between paid and unpaid work in Spain, Argentina and Chile.

Keywords: unpaid domestic work; time use; gender; comparative analysis

\begin{aligned} & \multicolumn{2}{c}{ Sumario } \\ & 1. Introducción 4. Base de datos y metodología \\ & 2. Fundamentos teóricos y conceptuales 5. Resultados \\ & 3. Contextos sociales: 6. Conclusiones \\ & la perspectiva macro Referencias bibliográficas \end{aligned}

\section{Introducción}

El análisis de las encuestas de usos del tiempo se ha mostrado como uno de los instrumentos más fecundos en la comprensión de las desigualdades por género, en especial en el análisis de las dimensiones del trabajo (remunerado y no remunerado) y en aquellas aproximaciones que abordan la distribución del tiempo, en las diferentes actividades, como un aspecto importante del bienestar de la población, reflejando desigualdades en el acceso a los recursos y afectando las oportunidades y capacidades (Aguirre, 2015; CEPAL, 2017a y 2016c; Batthyány, 2015a; Aguirre y Ferrari, 2014; Carrasco y Domínguez, 2014; Duran y Milosajevic, 2012; Antonoupolus, 2010; Eurostat, 2008).

Diversas investigaciones permiten constatar la tendencia a la convergencia entre mujeres y hombre en su dedicación a los trabajos: el aumento de la participación de las mujeres en el trabajo remunerado y una disminución en el trabajo no remunerado, con una mayor presencia de los hombres en el trabajo doméstico y de cuidados. Lenta convergencia en un contexto de continuidad y permanencia de la división sexual del trabajo que reproduce 
la asignación, casi exclusiva, de las tareas domésticas de carácter reproductivo y de cuidados a las mujeres (Kan et al., 2011; Bianchi et al., 2000; Gálvez et al., 2010; Kan y Gershuny, 2010; Sayer, 2010; Treas, 2010; Hook, 2006; Gershuny, 2000; Aguirre, 2009; Moreno, 2009; Marini y Shelton, 1993).

Así mismo, se constata que la presencia de las mujeres en el trabajo remunerado no ha ido acompañada de una participación equivalente de los varones en la reproducción doméstica ni de transformaciones en el reparto del trabajo doméstico y de cuidados que implicasen responsabilidades compartidas. Esta mayor carga de trabajo, y la consiguiente reducción del tiempo libre, del tiempo para si y de sus tiempos de participación social y política, limita las opciones de incorporarse al mercado laboral, acceder a puestos de trabajo más diversificados y obtener ingresos suficientes; en definitiva, menor bienestar y calidad de vida de las mujeres y el mantenimiento de las relaciones de poder y el orden de género (CEPAL, 2017b y 2016b; Ferrant et al., 2014; Aguirre et al., 2005; Calero et al., 2015).

Esta situación es aún más apremiante en aquellas mujeres con menores recursos para el empleo (nivel educativo y trayectorias laborales previas), con mayores demandas de cuidados de los hogares (presencia de personas menores, adultas mayores, enfermas o incapacitadas) y con menores posibilidades de derivar las necesidades de cuidados del hogar (a ámbitos extradomésticos, públicos o privados, o a redes de solidaridad) (Rodríguez, 2015).

Es indudable que el trabajo doméstico y de cuidados no remunerado es un factor indispensable que contribuye al bienestar de las personas, sus familias y las sociedades. Así pues, considerar las familias como proveedoras de bienestar y el papel central - aunque oculto - de una parte importante del trabajo realizado por las mujeres posibilitan identificar la división sexual del trabajo (la división entre trabajo remunerado y no remunerado) y el uso diferenciado del tiempo como el puntal de la subordinación económica, social y política de las mujeres (Aguirre, 2015; Ferrant et al., 2014; Carrasco y Domínguez, 2011; Francavilla et al., 2009; Folbre, 2008; Arriagada 2007).

El objetivo de este artículo es analizar los factores explicativos de la brecha de género en la distribución del trabajo no remunerado y de cuidados dentro de los hogares. Concretamente se analiza su distribución entre las personas principales del hogar a partir del análisis del uso del tiempo en España, Argentina y Chile.

De forma concreta, se constata cómo el trabajo doméstico y de cuidados realizado sin remuneración desde los hogares, uno de los componentes fundamentales de bienestar, es realizado fundamentalmente por las mujeres; en un contexto de substitución del modelo más tradicional de un solo proveedor (male breadwinner/housewife household) por el modelo de dos preceptores de ingresos (Díaz et al., 2015; García, 2012; Dema, 2006; Dunn y Skaggs, 2006). Y si ese es el modelo dominante, es de interés analizar cómo se han modificado las estrategias, en relación con las aportaciones en tiempos y trabajos de mujeres y hombres, dentro de los hogares. 
El estudio plantea un análisis comparativo entre Argentina, Chile y España ${ }^{1}$. Países que comparten elementos de desarrollo, pero que también presentan diferencias en sus arreglos de bienestar, valores de género y niveles de desigualdad, por lo que este análisis comparado incluye perspectivas acerca de las políticas de igualdad de oportunidades y sus alcances.

Ciertas investigadoras (Amarante y Rossel, 2017; Martínez, 2005) señalan que en América Latina la investigación comparativa sobre la distribución del trabajo doméstico y de cuidados es más reciente y fragmentada que en Estados Unidos y Europa. En los últimos años, el desarrollo de las encuestas de uso del tiempo en la mayoría de países latinoamericanos, y los estudios auspiciados por la Comisión Económica para América Latina y el Caribe (CEPAL), junto a los institutos de estadística nacionales, han permitido generar información relevante para evidenciar las desigualdades de género en el tiempo dedicado al trabajo doméstico y de cuidados no remunerado (Rico y Robles, 2016; Batthyány, 2015a; Calero et al., 2015; Rodríguez, 2015; Vaca-Trigo, 2015; Aguirre y Ferrari, 2015, 2014; Calderon, 2013; Duran y Milosajevic, 2012; Budlender, 2010; Espejo et al., 2010; Esquivel, 2010; Aguirre, 2009; Arriagada, 2007; Salvador, 2007).

Los resultados que se destacan en estos estudios son contundentes y se encuentran alineados con las evidencias de otras encuestas de uso del tiempo a nivel internacional (Rodríguez, 2015). Evidenciando la mayor carga global de trabajo de las mujeres, el aumento de la brecha de género y la incidencia de la estratificación socioeconómica (Gómez et al., 2017; CEPAL, 2017a, 2016c y 2016a; Batthyány, 2015a; Calero et al., 2015; Rodríguez, 2015; Calderon, 2013; Aguirre, 2009; Arrigada, 2007).

Por su parte, los trabajos empíricos comparativos en América Latina aún son débiles, escasos e incompletos. En esta línea se encuentran los trabajos de Amarante y Rossel (2017), con datos de Colombia, México, Perú y Uruguay; Campaña et al. (2017), con su estudio con los datos de usos del tiempo de México, Perú y Ecuador; Budlender (2010), con su trabajo de Argentina, Nicaragua, India, República de Corea, Sudáfrica y Tanzania; y Antonopoulos (2008), con las encuestas de uso del tiempo de Bolivia, Guatemala, México, Nicaragua y otros países de la OCDE.

Argentina y Chile, países que han realizado sus encuestas de usos del tiempo, con cobertura nacional, recientemente, se encuentran poco presentes en los estudios comparativos; y si lo están, es con fuentes de datos incompletas y antiguas. En el caso español, son más habituales los trabajos y estudios comparativos en el contexto de los países europeos (Moreno, 2015; Francavilla et al., 2013; Francavilla et al., 2009; Geist, 2005; Sevilla-Sanz et al., 2010; Eurostat, 2004).

1. Este artículo se ha elaborado en el contexto de la Red INCASI, proyecto europeo que ha recibido financiación del programa de investigación H2020 de la Unión Europea, Marie Skłodowska-Curie GA No 691004, coordinado por el Dr. Pedro López-Roldán. El artículo refleja la opinión de los autores y la Agencia no es responsable del uso que se pueda hacer de la información que contiene. 
Así, mediante el análisis comparativo de las encuestas del uso del tiempo en Argentina, Chile y España, este estudio identifica los principales factores que, tanto a nivel individual como contextual, están relacionados con la brecha de género en la asignación y distribución del trabajo no remunerado y de cuidados dentro de los hogares. Específicamente, se profundiza en las estrategias de aquellas parejas que se señalan como más igualitarias en el reparto de este trabajo, las parejas de doble ingreso, y se examina en qué medida estamos frente a una transformaciabajo remunerado y no remunerador las personas principales del hogar, n quparativos en el contexto de los paises s que se realizón en los modelos de usos de tiempo en términos de mayor igualdad. Esta aproximación posibilitará incidir en los determinantes de la división del trabajo doméstico y de cuidados entre mujeres y hombres.

\section{Fundamentos teóricos y conceptuales}

El propio concepto de trabajo doméstico y de cuidados no está exento de debate, con distintas conceptualizaciones y aproximaciones (Lamaute-Brisson, 2013; Esquivel, 2011; Albelda et al., 2009).

De forma concreta, se puede definir como una función social que integra actividades, bienes y relaciones (por lo general cara a cara) destinadas al bienestar cotidiano de las personas, e incluye la provisión de bienes básicos para la vida (alimentación, abrigo, higiene, acompañamiento), así como el apoyo y la transmisión de conocimientos, valores sociales y prácticas de crianza (Rico y Robles, 2016; Marco y Rico, 2013; Razavi 2012).

Con relación al trabajo de cuidados no remunerado, Ferrant et al. (2014) lo definen como todos realizados sin recibir remuneración; suministrados dentro de un hogar para sus miembros; e incluyendo el cuidado de personas (la actividad proporciona lo que es necesario para la salud, el bienestar, el mantenimiento y la protección de alguien o algo), el trabajo doméstico y el trabajo comunitario voluntario.

En este artículo lo denominaremos trabajo doméstico y de cuidados, siguiendo la aproximación de Bundlerder (2010), que distingue: el trabajo doméstico no remunerado para el hogar (ampliamente equivalente a tareas domésticas) y el trabajo de cuidados no remunerado a personas del hogar. Aunque diversos estudios señalan la necesidad de diferenciar el trabajo doméstico y el trabajo de cuidados, debido a su distinta naturaleza, significado y predictores (Knudsen y Waerness, 2008; Davis et al., 2007; Fuwa y Cohen, 2007; Mannino y Deutsch; 2007; Bartley et al., 2005; Bianchi y Raley; 2005; Coltrane y Adams, 2001), en este trabajo empírico se analizará en forma agregada dado el interés en profundizar en la brecha de género en la distribución del trabajo global no remunerado que se realiza desde los hogares, sin diferenciar su contenido. Explícitamente, se ha excluido el trabajo no remunerado para otros hogares, para la comunidad y el voluntario, fundamental también en las estrategias de subsistencia y de bienestar de los hogares. 
Tres han sido las perspectivas teóricas explicativas dominantes a nivel micro para dar cuenta de la división del trabajo doméstico y de cuidados no remunerado que se realiza desde los hogares: la disponibilidad de tiempo, los recursos relativos y los roles de género (Lachance-Grzela y Bouchard, 2010; Gupta y Ash, 2008; Gupta, 2006 y 2007; Fuwa, 2004; Parkman, 2004; Bittman et al., 2003; Bianchi et al., 2000; Greenstein, 2000; Coltrane, 2000; Katz, 1997).

La perspectiva de la disponibilidad de tiempo, basada en la teoría del capital humano y la teoría de la familia de Gary Becker, entiende la división del trabajo doméstico y de cuidados no remunerado como una asignación racional consecuencia de otras demandas sobre el tiempo de las personas. Supone un hogar formado por personas que maximizan una función de utilidad, sobre la base de un conjunto de preferencias comunes y unas restricciones de presupuesto común (Coverman, 1985). Las personas del hogar contribuyen, a las distintas actividades, de manera diferenciada según sus habilidades especializadas, productividad y beneficios esperados. Por tanto, el mayor tiempo dedicado por las mujeres al trabajo doméstico y de cuidados sería una consecuencia del mayor salario de los hombres, y de la especialización femenina en este trabajo. Por tanto, se constata que a mayor tiempo dedicado al trabajo remunerado, menor es la dedicación al trabajo doméstico y de cuidados; lo que explica la relación positiva entre las horas de trabajo en el empleo de las mujeres y el trabajo doméstico y de cuidados no remunerados de sus compañeros masculinos (Davis et al., 2007; Gershuny y Sullivan, 2003; Bianchi et al., 2000; Greenstein, 1996; Brines, 1994).

Si embargo, la mayoría de los estudios observan que la brecha de género en el tiempo de trabajo doméstico y de cuidados no se explica totalmente por este presupuesto: la relación entre ambos trabajos es más visible para las mujeres y ellas siempre, sea cual sea el tiempo dedicado al trabajo de mercado, dedican más tiempo al trabajo doméstico y de cuidados que los hombres (Bartley et al., 2005).

Por su parte, la perspectiva de los recursos relativos, o la teoría del intercambio económico, entiende el proceso como una forma de negociación entre los miembros de la pareja, en el que los ingresos, la educación y el prestigio ocupacional son recursos del poder de negociación de las personas (Pinto y Coltrane, 2009; Bernhardt et al., 2008; Knudsen y Waerness, 2008; Mannino y Deutsch, 2007; Fuwa, 2004).

En este enfoque, las personas tratarán de minimizar su tiempo dedicado a las tareas domésticas y de cuidados, usando cualquier ventaja de recursos para negociar, de mejor forma, su ausencia del trabajo doméstico y de cuidados (Brines, 1994; Deutsch et al., 1993; Coverman, 1985). Los estudios empíricos que toman los ingresos de cada uno de los miembros de la pareja como factor explicativo de la distribución de los trabajos confirman estos supuestos (Sevilla-Sanz et al., 2010; Dema, 2006; Bianchi et al., 2000). Esta perspectiva se amplía con incorporación de la teoría de Blumberg (Wallace, 1989) de la desigualdad de género, que afirma que las mujeres con mayores ingresos salariales deberían tener más poder en la negociación y, por lo tanto, compartir 
las tareas domésticas de manera más equitativa. Por tanto, cuanto menor es la brecha de ingresos entre las dos personas de la pareja, más igualitaria es la división del trabajo doméstico y de cuidados (Bittman et al., 2003; Davis et al., 2007). Los resultados empíricos sobre el efecto del nivel educativo y el prestigio ocupacional, así como los aportes relativos de ingresos de las mujeres, no son concluyentes y muestran una alta diversidad e inconsistencia en contextos diversos: (Lachance-Grzela y Bouchard, 2010; Knudsen y Waerness 2008; Fuwa, 2004; Goldscheider y Waite, 1991). Además, junto a ello, dicha aproximación ha recibido severas críticas por la invisibilidad a las relaciones de género, normas y percepciones sociales que pueden determinar la capacidad de negociación de mujeres y hombres en el interior del hogar (Katz, 1997).

Las relaciones de género es lo que plantea la perspectiva de la visualización —exhibición - de los roles de género. Según esta perspectiva, las mujeres y los hombres son socializados para adaptarse a los roles construidos socialmente; $y$ la división sexual en los trabajos es tanto una de las consecuencias como una construcción cotidiana de género en las relaciones con el otro sexo. Así pues, un comportamiento condicionado por las expectativas y las normas sociales que unas personas tienen del comportamiento de otras (Killewald y Gough, 2010; Gupta y Ash, 2008; Davis et al., 2007; Gupta, 2006 y 2007; Fuwa, 2004; Brines, 1994). En este sentido, diversos estudios constatan una relación positiva entre las actitudes y los roles de género igualitarios de la pareja femenina y/o la pareja masculina y una división más equitativa del trabajo doméstico y de cuidados (Kan y Gershuny, 2010; Sevilla-Sanz et al., 2010; Treas y Drobnic, 2010; Knudsen y Waerness, 2008; Davis et al., 2007; Fuwa, 2004; Parkman, 2004; Greenstein, 1996).

En este sentido, por una parte, las mujeres asumen el trabajo doméstico y de cuidados no solo como respuesta a las necesidades de bienestar de las personas del hogar, sino también como una forma de construirse apropiadamente como mujeres. Por otra, para los hombres el empleo puede ayudarles a construirse como hombres liberándolos de participar en las tareas domésticas y familiares (Bianchi et al., 2000; Spitze, 1988). Esta hipótesis explicativa puede explicar situaciones que serían incongruentes para otras perspectivas teóricas: la baja implicación de los hombres, económicamente dependientes, en tareas domésticas; la participación más alta de las mujeres en el trabajo doméstico; así como los resultados anómalos con respecto a los factores de la disponibilidad de tiempo y los recursos relativos.

A la luz de los principales resultados de los estudios realizados en Argentina, Chile y España, se puede afirmar que los fundamentos de las teorías presentadas son complementarios entre sí. Las evidencias empíricas muestran que el modelo de trabajo de las mujeres es el de la doble carga de trabajo (dentro y fuera del hogar), y revelan el poder explicativo de aquellos factores que sostiene la teoría de los recursos relativos: los ingresos, el nivel educativo y el tipo de ocupación. Por su parte, la división más igualitaria del trabajo que muestran las parejas de doble ingreso revelan la importancia de los procesos y las capacidades de negociación. Y finalmente, los resultados constatan, tal como sostiene la 
hipótesis de la disponibilidad de tiempo, el menor tiempo dedicado a las tareas domésticas y de cuidados de las personas trabajadoras a tiempo completo; y la reducción de la participación en las tareas no remuneradas, tanto de mujeres como de hombres, cuando el otro miembro de la pareja tiene más tiempo disponible (Domínguez, 2012; Balcells, 2009: González-López, 2001).

Ahora bien, ciertos resultados también evidencian que la situación en el empleo de la mujer y sus recursos relativos no son suficientes para explicar la segregación por género del trabajo doméstico y de cuidados, y señalan la validez explicativa de los factores de socialización y roles de género (Moreno, 2015; Carrasco y Domínguez, 2011). Normas que están arraigadas en la sociedad española y en los dos países latinoamericanos que nos ocupan en este trabajo (Sevilla et al., 2010).

\section{Contextos sociales: la perspectiva macro}

A partir del año 2000, surgen estudios que se centran en la relevancia de los factores macrosociales en la comprensión de la división del trabajo doméstico y de cuidados no remunerados en el hogar. Especialmente estudios comparativos, que analizan elementos macro que pueden incidir en el sentido y la magnitud de los efectos de las características individuales, su efecto (re)configurador en la división sexual del trabajo, así como la incidencia que pueden tener las políticas sociales. El presupuesto que subyace es que estos factores, además de una aproximación a la sociedad que se estudia, lo son también a la ideología y las actitudes de género (Amarante y Rossel, 2017; Yu y Xie, 2014; LachanceGrzela y Bouchard, 2010; Hook, 2006; Fuwa 2004).

En este sentido, operativizan el contexto social en cinco dimensiones: 1) el nivel de igualdad de género; 2) el desarrollo económico; 3) la estructura social y demográfica; 4) las políticas estatales relacionadas con la igualdad de género; y 5) las normas culturales (Batthyány, 2015b; Blofield y Martínez, 2014; Lewis, 2010; Arriagada, 2007; Fuwa, 2004).

De forma particular, Geist (2005), a partir de datos de la International Social Survey Program (GESIS-Leibniz Institute for the Social Sciences, Germany, 2017), investiga la distribución del trabajo no remunerado dentro del hogar en 10 países, destacando la relevancia del régimen de bienestar. Así pues, señala que en regímenes con un estado del bienestar conservador, que se caracterizan por mantener los roles de género tradicionales, se mantiene una marcada división sexual del trabajo no remunerado realizado desde los hogares (como Japón, Italia y Austria). En cambio, regímenes socialdemócratas (como Suecia y Noruega), que defienden activamente la equidad de género, muestran una distribución del trabajo doméstico y de cuidados más equitativa. Por su parte, en aquellos regímenes que se pueden caracterizar de liberales (como Australia, Canadá y Estados Unidos), los resultados son más heterogéneos.

Sayer (2010), por su parte, destaca la importancia de los cambios acaecidos con la segunda transición demográfica en la reducción de la brecha de género en el tiempo de trabajo doméstico y de cuidados: el acceso a la educación 
Tabla 1. Índice de la Brecha de Género y subíndices por dimensiones. Puntuación y posición en el ranking de países, 2017

\begin{tabular}{|c|c|c|c|c|c|c|}
\hline \multirow[b]{2}{*}{$\begin{array}{l}\text { Puntuación global brecha género } \\
\text { Indices por dimensiones }\end{array}$} & \multicolumn{2}{|c|}{ Argentina } & \multicolumn{2}{|c|}{ Chile } & \multicolumn{2}{|c|}{ España } \\
\hline & 0,732 & (34) & 0,704 & (63) & 0,746 & (24) \\
\hline Participación y oportunidad económica & 0,596 & (111) & 0,573 & (117) & 0,657 & (81) \\
\hline Logro educativo & 0,998 & $(44)$ & 0,999 & $(39)$ & 0,998 & (45) \\
\hline Salud y supervivencia & 0,980 & (1) & 0,978 & $(47)$ & 0,973 & (81) \\
\hline Empoderamiento Político & 0,356 & (21) & 0,266 & (36) & 0,354 & (22) \\
\hline
\end{tabular}

En los índices el valor máximo es 1 (paridad) y el mínimo es 0 (disparidad).

Entre paréntesis posición en el ranking de países, total de 144 países.

Fuente: Global Gender Gap Report 2017, Fórum Económico y Mundial.

superior y el incremento de la participación laboral de las mujeres; así como la consolidación de los derechos sociales de igualdad de oportunidades.

A la luz de estos estudios que focalizan su atención en los aspectos macrosociales, es necesario dar cuenta de los países analizados, a fin de constatar las posibles influencias de estos factores, junto a las variables individuales, en la distribución del trabajo doméstico y de cuidados en el hogar.

Con relación a la igualdad de género - como aproximación a las oportunidades, desigualdades y discriminaciones por razón de género en ámbitos económicos, laborales, sociales y políticos-, se constata que en aquellos países con un nivel superior en la brecha de género, el papel de la mujer se restringe a las funciones domésticas y reproductivas (Ferrant et al., 2014; Rizavi y Sofer, 2010).

En la tabla 1, se presentan los datos de los tres países analizados tomando como referencia el Índice de Brecha de Género del Fórum Económico Mundial $(2017)^{2}$.

Respecto a la estructura demográfica, en los tres países se observa un descenso sostenido del ritmo de crecimiento de la población y una tendencia al envejecimiento, con mayor incidencia y alcance temporal en España. La proporción de mayores de 65 años, en 2017, representa el 14,4\%, el 11,4\% y el 18,7\% en Argentina, Chile y España respectivamente. Es destacable la aceleración de estos cambios en Chile, que pasa del 6,6\% en 2002 al 11,4\% en 2017.

La baja tasa de natalidad de España, 1,33 hijos de promedio, frente a Chile, 1,94, y Argentina, con 2,29 hijos, así como la postergación de la edad del nacimiento de los hijos (32 años en el caso español y de aproximadamente 28 años en Argentina y Chile), señalan las diferencias en las necesidades de cuidados de estos países. En este aspecto, cabe también destacar que la esperanza de vida saludable de las mujeres es siempre más alta que la de los hombres: 70,2, 72,3 y 74 en Argentina, Chile y España respectivamente.

2. En los datos que se presentan en este apartado, se ha tomado como fuente los censos de población nacionales, así como los indicadores del Fórum Económico Mundial, correspondientes a 2017. 
Lo anterior trae como correlato el aumento de los hogares unipersonales y unas necesidades de cuidado hasta ahora no consideradas en los esquemas de protección y las políticas, especialmente en los países latinoamericanos.

En relación al logro educativo, el índice refleja una mayor igualdad en los tres países, y el acceso generalizado de las mujeres a los niveles educativos superiores.

La incorporación de la mujer en el mercado laboral y su independencia económica y financiera otorgan a las mujeres mayor capacidad negociadora en una división igualitaria del trabajo no remunerado (Amarante y Rossel, 2017; Yu y Xie, 2014; Lachance-Grzela y Bouchard, 2010; Fuwa 2004). Por tanto, la brecha de género en lo que respecta a la participación, los salarios y la presencia de las mujeres en categorías socioprofesionales altas permite incidir en este aspecto clave.

$\mathrm{Al}$ respecto, se observa una menor participación laboral femenina en los países latinoamericanos, y en condiciones desiguales, tal como muestra el índice de brecha de genero en este ámbito: 0,59, 0,57 y 0,65 en Argentina, Chile y España respectivamente. Mientras que la participación laboral masculina muestra pocas variaciones entre los países $(81,7 \%, 80,1 \%$ y 86\%, en Argentina, Chile y España respectivamente), la participación femenina es menor a la masculina: 68,9\% en España, 56,5\% en Argentina y del 57,9\% en Chile. En estos dos últimos países, es especialmente relevante que del total de mujeres ocupadas, el 50,7\% en Argentina y el 40,6\% en Chile lo hagan en el sector informal (aunque este fenómeno no es exclusivo de las mujeres, también el $50,8 \%$ de los hombres argentinos y el 36,5\% de los chilenos).

Si bien la participación laboral de la mujer es más alta en España, si se fija la atención en otros aspectos que inciden en las condiciones de esta participación, se visibiliza la desigualdad en el mercado laboral.

En relación a las diferencias salariales por genero por un empleo similar, estas se mantienen bastante análogas en los tres países (0,48 en Argentina, 0,53 en Chile y 0,51 en España;), y ocupan posiciones bastante desfavorecidas en cuanto a la disparidad. De forma concreta, en Chile, el Ministerio de Desarrollo Social constata que en el período 2006-2015 las mujeres de 15 años y más percibieron menos del 38\% del total del ingreso y un ingreso mediano mensual un $25 \%$ inferior, pasando de $33,0 \%$ a $37,7 \%$. Por su parte, en Argentina, estas brechas varían a medida que la población adquiere mayor educación, de modo que las diferencias salariales son del $20 \%$ entre las personas trabajadoras del nivel profesional, de $23 \%$ del nivel técnico y de $18 \%$ en el nivel operativo; y aumentan al 36\% entre las personas sin calificación.

Lo anterior también se observa si se analizan los datos de la presencia de las mujeres en las categorías socioprofesionales más cualificadas. En la categoría de dirección, legislación y alta cualificación, las mujeres son el 31,2\% y el 30,6\% en España y Argentina respectivamente; en Chile desciende al 27\%. En este último país, también en la categoría de profesionales y personal técnico, el porcentaje de las mujeres es sensiblemente inferior a los hombres $(46,9 \%)$; siendo más paritario en España y Argentina. 
Por último, señalar que en los tres países la proporción de mujeres desocupadas es mayor, así como las que trabajan una jornada parcial (menos de 30 horas) de manera involuntaria. El porcentaje de mujeres que están ocupadas a tiempo parcial en Argentina es del 41,5\%, en Chile del 34,1\% y en España del $32,1 \%$; mientras que los hombres lo están en el $16,8 \%$, el $19,8 \%$ y el $16,1 \%$ respectivamente. La brecha de género al respecto es visible, de forma grave en el caso de las mujeres argentinas.

En síntesis, las mujeres que acceden al mercado de trabajo no lo hacen en igualdad de condiciones que los varones, no ocupan puestos directivos de forma equitativa y no perciben los mismos salarios por el mismo trabajo y la misma formación. Las responsabilidades familiares y actividades domésticas propias del trabajo reproductivo determinan y condicionan esta inserción laboral.

Con relación al trabajo no remunerado y de cuidados, las encuestas nacionales del uso del tiempo constatan que las mujeres registran mayor participación y tiempo que los hombres. La brecha en tiempo indica que las mujeres trabajan entre dos y tres horas más que los hombres en trabajo no remunerado en un día tipo (3, 1,94 y 2,13 horas más al día en Argentina, Chile y España respectivamente) (INE, 2011; INDEC, 2014; INE-Chile, 2016a).

Es justamente esta distribución desigual la que, entre otros elementos, restringe la participación de las mujeres en el mercado laboral, condicionada por la adscripción de las mujeres al trabajo doméstico y de cuidados. Así pues, si tomamos como ejemplo el caso argentino, se observa que la tasa de actividad femenina disminuye a medida que aumenta el número de menores en el hogar, y esto sucede de forma simultánea con el aumento de la tasa de actividad masculina. La brecha de género pasa del $27,4 \%$, cuando no existen menores en el hogar, al 38\% cuando hay un menor solamente, y a 50\% cuando hay dos o más menores.

Si el análisis se realiza tomando aquellos indicadores referentes a la ideología y las actitudes hacia los roles de género y los estereotipos, en la tabla 2 se presentan los resultados que proporciona la Encuesta Mundial sobre Valores Sociales (WVS) (Institute for Comparative Survey Research, 2017), implementada de forma homogénea en los tres países.

Los resultados muestran que, en comparación con los otros países, en España hay una mayor aceptación de valores igualitarios de género, especialmente en aquellas afirmaciones que reflejan la opinión sobre la igualdad de las mujeres en su participación en el mercado laboral y su independencia económica (las tres primeras afirmaciones). Así mismo, se destaca Chile por el mantenimiento de valores de género no igualitarios: un $41,9 \%$ no rechaza la preferencia por los hombres si hay escasez de lugares de trabajo; un 66,2\% opina que puede ocasionar problemas el hecho de que la mujer aporte más ingresos que el hombre; $y$ un $37,8 \%$ coincide con la afirmación que cuando una madre tiene un trabajo remunerado, los hijos/as sufren.

En cuanto a la legislación, y otras medidas legales relacionadas con el concepto de «equidad de género», interesa profundizar si el Estado reconoce la 
Tabla 2. Opiniones sobre ciertos aspectos relacionados con la ideología de género, según países. World Value Survey (porcentajes de repuestas de acuerdo y neutras a la sentencia)

\begin{tabular}{|c|c|c|c|}
\hline & Argentina & Chile & España \\
\hline $\begin{array}{l}\text { Cuando los puestos de trabajo escasean, los hombres deberían } \\
\text { tener más derecho que las mujeres a un puesto de trabajo }\end{array}$ & 29,5 & 41,9 & 17,5 \\
\hline $\begin{array}{l}\text { Si una mujer gana más que su marido es casi seguro que creará } \\
\text { problemas }\end{array}$ & 46,0 & 66,2 & 25,0 \\
\hline Cuando una madre tiene un trabajo remunerado, los hijos sufren & - & 37,8 & 28,5 \\
\hline $\begin{array}{l}\text { En general, los hombres son mejores líderes políticos que las } \\
\text { mujeres }\end{array}$ & 27,5 & 28,2 & 11,5 \\
\hline $\begin{array}{l}\text { Una educación universitaria es más importante para un muchacho } \\
\text { que para una muchacha }\end{array}$ & 16,6 & 20,9 & 11,7 \\
\hline $\begin{array}{l}\text { Ser un ama de casa es casi tan gratificante como tener un trabajo } \\
\text { remunerado }\end{array}$ & 54,2 & 44,4 & 49,5 \\
\hline
\end{tabular}

Argentina, 2013; Chile, 2012, y España, 2011. Institute for Comparative Survey Research (2017).

Fuente: elaboración propia a partir de las encuestas correspondientes a la sexta oleada de la World Value Survey.

existencia de desigualdades que requieren la adopción de medidas diferenciadas en función del sexo para garantizar la igualdad y el respeto de los derechos humanos (Sayer, 2010). Como indicadores indirectos, para medir el grado de promoción de la igualdad de género en la legislación nacional, se consideran aspectos tales como: la existencia de legislación sobre la violencia contra la mujer (existente en los tres países) y la existencia de legislación o de medidas por las que se establezcan sistemas de cupos para la participación política de las mujeres.

Respecto a este último aspecto, el empoderamiento político, los datos de la tabla 1 muestran el bajo índice y la mayor disparidad entre mujeres y hombres en los tres países: el 0,26 de Chile y el 0,35 de Argentina y España.

Concretamente, en los tres países existe una ley de cuotas en las elecciones: del $40 \%$ en España y Chile (en este último solo a nivel parlamentario) y del $30 \%$ en Argentina. Ahora bien, la paridad en la representación política está lejos de alcanzarse ya que el porcentaje de mujeres en el Parlamento es del $39,1 \%$ y del 38,9\% en España y Argentina, frente al 15,8\% en Chile (si bien los porcentajes se intercambian entre estos dos países cuando se analiza el porcentaje de mujeres en cargos de ministras: el 17,4\% en Argentina, el 30,8\% en Chile y el 38,5\% en España).

Por último, se considera relevante centrar la mirada en el análisis de políticas, dado que diversos estudios han señalado que, en aquellos países que implementan políticas laborales basadas en la igualdad de oportunidades y políticas sociales de apoyo al cuidado (de permisos parentales, tiempos de trabajo flexibles, trabajo remunerado en casa), se constata una mayor convergencia en el uso del tiempo de mujeres y hombres (Moreno, 2015; Kan et al., 2011; Gálvez et al., 2010; Lachance-Grzela y Bouchard, 2010; 
Treas y Drobnic, 2010; Hook, 2006; Crompton et al., 2005; Gershuny y Sullivan, 2003).

Por el contrario, en países donde persiste el modelo de hombre sostenedor del hogar, las desigualdades de género en la distribución del trabajo doméstico y de cuidados es mayor. Este es el caso de los tres países analizados, aunque bajo realidades muy distintas entre ellos si retomamos las diferencias en la participación de las mujeres en el mercado laboral y las ideologías de género.

Tal como señala Martínez (2005), Argentina y Chile se situarían en un régimen de bienestar «liberal de proveedor único», que se denominará como "mercado-céntrico», donde se ha registrado un desplazamiento acelerado y radical desde el Estado hacia la prestación privada de servicios, en particular de salud, educación y pensiones.

Más específicamente, en cuanto al modelo de bienestar en Argentina, se produjo el traspaso de un régimen político con intervención estatal en el desarrollo económico y social del país (2003-2015) a un modelo emparentado con la tradición residual liberal (2015-2019) (Filgueira, 2007). En consecuencia, desde principios de siglo se aplicó un modelo de protección social orientado tanto a los sectores urbanos, con cobertura para los sectores del empleo formal, como a los trabajadores rurales e informales, a partir de programas específicos. Aunque dicha estrategia no llegó a cubrir el total de la población, a partir de 2015 se produjo cierto retroceso: los ejes del modelo se reorientaron a reducir el empleo formal - y con ello la cobertura social de los trabajadores desempleados-; a descentralizar la gestión de los programas sociales; y a reducir o eliminar varios de estos, delegando en el mercado gran parte de las funciones sociales. $\mathrm{Al}$ respecto, Chile tiene una arquitectura de protección social basada en un esquema de tipo liberal, donde el Estado actúa en forma residual a los arreglos familiares y de mercado (Midaglia y Antía, 2017). Filgueira (2007) definió el esquema de protección del país bajo la categoría de universalismo estratificado, con amplia cobertura de población y riesgos sociales, aunque altamente diferenciado de acuerdo con las condiciones de incorporación de la población al mercado de trabajo.

Hoy en día el modelo en Chile se asocia a un modelo estatal productivista, que se concentra en la formación de capital humano. Con un proceso de expansión restrictivo de garantías sociales que establece parámetros de calidad en las prestaciones que se dirigen a los sectores tradicionalmente protegidos (los trabajadores formales) a nuevos segmentos (los trabajadores informales y desempleados) (Midaglia y Antía, 2017).

La evolución del sistema de bienestar, tanto en Chile como en Argentina, va cambiando de acuerdo con la orientación ideológica de los gobiernos en el poder. En consecuencia, las políticas están orientadas de manera alternada a ampliar y/o a reducir las coberturas de riesgos sociales y a mejorar o empeorar las condiciones de la fuerza laboral para participar del mercado de trabajo Así, si bien en ciertos momentos las políticas se encaminan hacia un mayor reconocimiento de derechos, introduciendo algunos ajustes para moderar las desigualdades generadas, a partir de un conjunto de reformas orientadas a 
extender la cobertura hacia sectores de la población que antes no accedía a la protección social, en otros momentos ese camino se desanda.

En el caso español, Moreno (2007) destaca el familismo como referente normativo subyacente en el imaginario social colectivo ${ }^{3}$ y en la práctica institucional, que da lugar a un modelo de políticas familiares y sociales escasamente desarrollado, a la permanencia del modelo familiar "medio» de varón sustentador, a y la desigual participación de hombres y mujeres en las tareas domésticas y en el cuidado de los hijos. Ello acompañado por una reducida intervención pública, en materia familiar, y con la concepción de que los problemas derivados del empleo y la familia son un asunto privado. Aunque en los últimos años se han desarrollado políticas destinadas a favorecer el empleo femenino (tales como ampliación de permisos de maternidad y paternidad y la concesión de ayudas económicas por hijos dirigidas a las madres trabajadoras), estas han tenido que coexistir con la escasa flexibilidad del mercado laboral y el peso de la cultura familiarista.

El desarrollo de estas políticas sociales y de igualdad se han aplicado de la mano de una visión política socialdemócrata y de un importante proceso de europeización, que instala en la agenda tanto políticas de promoción del empleo femenino como políticas de cuidados (servicios, beneficios y permisos parentales) (Salazar, 2016; Lombardo y León, 2014).

Sin embargo, las consecuencias de la crisis económica (visible claramente a partir de 2010) tuvieron como respuesta la «austeridad» en política económica (reducción de salarios y del gasto público) y el ralentizamiento de las políticas de igualdad, en el marco de un proyecto político neoliberal, con el predominio de políticas de igualdad de oportunidades (Salazar, 2016; Bettio et al., 2012; Karamessini y Rubery, 2014).

España se sitúa en el modelo de régimen mediterráneo, caracterizado por el papel central de la familia, la escasa red de servicios públicos y la baja presencia de los hombres en la corresponsabilidad del trabajo doméstico y de cuidados (Kan et al., 2011; Gálvez et al., 2010). Aunque esta caracterización también podría ser válidas para Argentina y Chile.

En conclusión se puede afirmar que los tres países comparten una fuerte tradición familiarista, escasez de los servicios públicos de cuidados y traspaso al mercado, en especial en los países latinoamericanos; con una baja, y en condiciones desfavorables, participación laboral de la mujer y una ausencia del hombre del trabajo doméstico y de cuidados. Así mismo se observa un mantenimiento de una ideología de género, fuertemente arraigada en Argentina y Chile, que adscribe a la mujer al ámbito doméstico y familiar.

Con relación a la interacción entre factores individuales y contextuales, podemos afirmar que en aquellos países que se consideran menos igualitarios, Chile y Argentina en nuestro caso, las características individuales de las mujeres

3. Según la autora, en lo que se refiere a la percepción social de la conciliación y las actitudes y valores de las españolas ante el empleo y la familia, los estudios realizados confirman que los rasgos culturales asociados con el rol social de madre, esposa y trabajadora asignan un papel ambivalente a las mujeres ante el mercado laboral y el escenario familiar. 
tendrán un mayor impacto en la brecha de género en su participación y distribución del trabajo doméstico y de cuidados. Ello porque los valores culturales y las normas sociales, la socialización de roles de género, tienen un mayor peso en la distribución del trabajo no remunerado del hogar. Por su parte, en España donde la incorporación de la mujer en el mercado laboral y la igualdad de género es mayor, los cambios sociales en los roles de género inciden más que los procesos de negociación de la pareja en la distribución más equitativa del trabajo doméstico y de cuidados.

\section{Base de datos y metodología}

El trabajo empírico del estudio se desarrolló a partir de las encuestas de uso del tiempo de los tres países analizados.

En España, los últimos datos disponibles corresponden a la Encuesta de Empleo del Tiempo del 2010 (EET, 2010), realizada por el Instituto Nacional de Estadística (INE) siguiendo las directrices de las encuestas armonizadas europeas. En el caso Argentino, el Instituto Nacional de Estadística y Censos (INDEC) implementó por primera vez, en 2013 con cobertura nacional urbana, un módulo acotado, el Módulo de Trabajo no Remunerado y Uso del Tiempo (TNR, 2013), aplicado en la Encuesta Anual de Hogares Urbanos (EAHU). En 2015, se aplica, en el territorio nacional chileno, la primera Encuesta sobre el Uso del Tiempo (ENUT, 2015), realizada por el Instituto Nacional de Estadísticas (INE-Chile).

Las tres encuestas tienen como objetivo obtener información sobre el uso del tiempo de la población (de 12 y más años en Chile; de 18 y más en Argentina, y a partir de 10 años en España), respecto a actividades de trabajo realizadas en el mercado, el trabajo no remunerado y las actividades personales. Aunque un estudio sobre la utilización del tiempo de las persona puede cubrir multitud de necesidades de información, estas operaciones estadísticas permiten visibilizar la carga global de trabajo de la población a través de la medición del tiempo que se dedica al trabajo en la ocupación y sus actividades conexas y al trabajo no remunerado; así como cuantificar la magnitud y evidenciar las desigualdades de género, y de otras variables de clasificación; y contribuir a la formulación de políticas familiares y de igualdad entre géneros (INDEC, 2014; INE-Chile, 2016a; INE, 2011).

Con relación a los instrumentos de recogida de información, cabe señalar que son ejemplo de las alternativas metodológicas existentes: la EET-2010 española se realiza a partir de un diario de actividades (agenda cronológica) que registra lo que las personas hacen durante las 24 horas de un día (laborable más un sábado o festivo), en fracciones de 10 minutos. En la TRN-2013 de Argentina y la ENUT-2015 de Chile, la recogida de la información se realiza a partir de un listado de actividades (cuestionario estructurado en base a una selección de actividades de interés) donde se recoge si se ha realizado la actividad y el tiempo destinado (habitualmente en el día de ayer). En ambas encuestas, se utilizó como listado de actividades predefinidas la Clasificación de 
Actividades de Uso del Tiempo para América Latina y el Caribe (CAUTAL), que toma como referencia la Clasificación Internacional de Actividades para Estadísticas sobre Uso del Tiempo (ICATUS), empleada en la encuesta española (CEPAL/INEGI/INMUJERES/ONU-Mujeres, 2016).

En la operación estadística chilena, el uso del listado permite estructurar la encuesta relevando información sobre el conjunto de actividades definidas, organizadas en bloques temáticos: trabajo en la ocupación y desplazamientos; búsqueda de empleo; producción de bienes para el autoconsumo del hogar; cuidados no remunerados a miembros del hogar; trabajo no remunerado en quehaceres del propio hogar; trabajo no remunerado para otros hogares, para la comunidad y trabajo voluntario no remunerado; cuidados personales y necesidades fisiológicas; actividades de educación y aprendizaje; ocio y vida social.

En el caso argentino, además de no ser un cuestionario específico sino un módulo añadido a una encuesta regular, donde se incluyen las preguntas del trabajo remunerado, tiene la limitación de incluir un listado de actividades de trabajo no remunerado restringido. Un primer bloque está dedicado al trabajo doméstico y de cuidados no remunerado que considera los quehaceres domésticos; las actividades de cuidado de niños, enfermos o adultos mayores miembros del hogar; y las actividades dedicadas al apoyo escolar y/o de aprendizaje a miembros del hogar. En este caso, en las preguntas que incluían más de una tarea (las referidas a las tareas domésticas y a las tareas de cuidado), cuando la persona entrevistada hubiera realizado más de una actividad en forma simultánea, se pidió que contestara por aquella que identificara como principal. De manera que el módulo de trabajo no remunerado capta simultaneidad -implícitamente- entre los grupos de tareas de las tres preguntas, pero no entre las tareas de cada grupo (INDEC, 2014; Rodríguez, 2015). Además, en la encuesta argentina, se establece la condición de haber dedicado por lo menos una hora a la actividad para considerar que se realizó, así se elimina a las personas, fundamentalmente varones, que dedicaron menos tiempo, pudiendo sobreestimar su tiempo de trabajo no remunerado.

La EET 2010 de España recoge información mediante un diario de actividades a partir de un listado armonizado a la propuesta de Eurostat en sus directrices de 2008. La clasificación de las actividades se ha divido en 10 grupos: cuidados personales; trabajo remunerado; estudios; hogar y familia; trabajo; voluntario y reuniones; vida social y diversión; deportes y actividades al aire libre; aficiones e informática; medios de comunicación; trayectos y empleo del tiempo no especificado.

La diversidad de procedimientos metodológicos en las encuestas de uso del tiempo y las posibles discrepancias en los resultados han sido estudiadas ampliamente en diversos trabajos (Carrasco y Domínguez, 2014; Schulz y Grunow, 2011; Kan y Pudney, 2008; Bonke, 2005; Kitterod y Lyngstad, 2005; Juster et al., 2003; Press y Townsley, 1998; Marini y Shelton, 1993). Los resultados son muy dispares según el contexto, las características sociales y el tipo de actividad. La mayoría de los estudios señalan que los diarios ofrecen una información más fiable, completa y con mayores posibilidades de análisis; 
y que las magnitudes del tiempo dedicado a trabajo doméstico y de cuidados obtenidos a partir del cuestionario son mayores que las de los diarios en aquellas actividades que se realizan con más frecuencia, sobreestimándose la participación de las personas que realizan una mayor cantidad de trabajo. Sin embargo, se señala que las preguntas directas sobre el reparto y la distribución del tiempo doméstico y de cuidados no remunerado proporcionan resultados similares sea cual sea la forma de medición.

\subsection{Datos}

Ya que el interés es estudiar la dedicación horaria al trabajo doméstico y de cuidados y su distribución entre mujeres y hombres del hogar, se justifica el estudio comparativo entre los tres países. Tanto las aportaciones de los estudios que comparan los resultados obtenidos según diversas opciones metodológicas en las encuestas de usos del tiempo; así como la constatación que los resultados de las encuestas argentina y chilena son contundentes y se encuentran alineados, en términos generales, con las evidencias de otras encuestas de uso del tiempo a nivel internacional, se considera oportuno analizar las tres encuestas de usos del tiempo, de forma comparativa.

En ese sentido y para fortalecer la comparabilidad de las encuestas, el análisis se focalizará en la brecha de género en la distribución del trabajo doméstico y de cuidados entre los dos miembros principales de la pareja, como forma de controlar los efectos que pudieran tener los instrumentos de recogida de la información y asegurar su comparabilidad. Por ello, la variable dependiente es la contribución de cada miembro de la pareja al tiempo total que se destina en el hogar por las dos personas principales ${ }^{4}$ : calculando primero el total de tiempo que las dos personas principales, femenina y masculina ${ }^{5}$, dedican al hogar, y después la proporción que realiza cada uno de sus miembros.

Para facilitar la lectura del dato se ha multiplicado por 100, por lo que cuando tome el valor de 50 significará un reparto equitativo entre los dos miembros principales del hogar. En cambio, se aproximará a 0 o a 100 cuando mayor sea la desigualdad: 100 cuando es la mujer la que realiza la totalidad del trabajo doméstico y de cuidados.

Además, siguiendo la estrategia propuesta en otros análisis sobre el uso del tiempo (INE-Chile, 2016a), al disponer de la información de un día laborable o de un día sábado-domingo, se ha construido el tiempo dedicado a los distintos trabajos en un día tipo de la semana: la suma ponderada del tiempo, con proporciones de 5/7 para el tiempo destinado en día de semana y 2/7 para el tiempo destinado en el fin de semana.

4. Dado que nuestro interés es la distribución de los trabajos entre las dos personas principales del hogar, se excluyen los tiempos de trabajo remunerado y de trabajo doméstico y familiar realizado por otros miembros del hogar.

5. En el caso de las encuestas de Argentina y Chile, las personas principales del hogar son las que se identifican en el cuestionario jefe(a) del hogar y su cónyuge. En el caso de la encuesta de España, se identifican según la edad y el parentesco con el resto de los miembros del hogar. 
Tabla 3. Descripción de la muestra

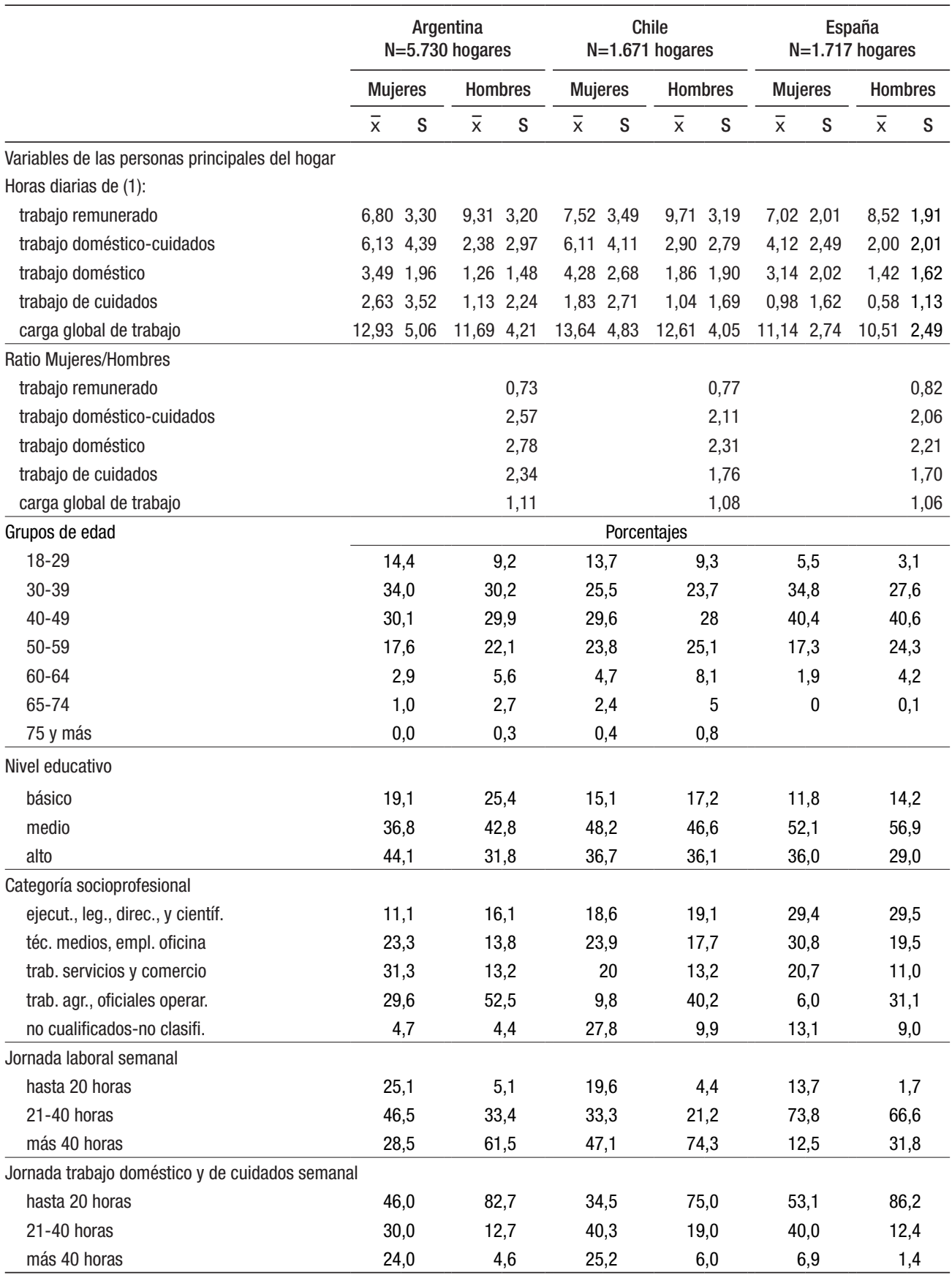

(continúa) 
Tabla 3. Descripción de la muestra (continuación)

\begin{tabular}{|c|c|c|c|c|c|c|c|}
\hline & \multicolumn{2}{|c|}{$\begin{array}{c}\text { Argentina } \\
\mathrm{N}=5.730 \text { hogares }\end{array}$} & \multicolumn{2}{|c|}{$\begin{array}{l}\text { Chile } \\
\mathrm{N}=1.671 \text { hogares }\end{array}$} & \multicolumn{3}{|c|}{$\begin{array}{c}\text { España } \\
\mathrm{N}=1.717 \text { hogares }\end{array}$} \\
\hline & Mujeres & Hombres & Mujeres & Hombres & Mujeres & \multicolumn{2}{|c|}{ Hombres } \\
\hline & $\bar{x}$ & $\bar{x}$ & $\bar{x}$ & $\bar{x}$ & $\bar{x}$ & $\bar{x}$ & $\mathrm{~S}$ \\
\hline \multicolumn{8}{|l|}{ Variables del hogar } \\
\hline $\begin{array}{l}\text { Horas diarias totales de trabajo doméstico } \\
\text { y de cuidados realizadas por las personas } \\
\text { principales del hogar (1) }\end{array}$ & $8,516,19$ & & $9,01 \quad 5,61$ & & $6,123,55$ & & \\
\hline $\begin{array}{l}\text { Ratio de trabajo doméstico y de cuidados } \\
\text { mujeres/total }\end{array}$ & $0,76 \quad 0,22$ & & $0,69 \quad 0,21$ & & $0,69 \quad 0,24$ & & \\
\hline $\begin{array}{l}\text { Ratio aporte de los ingresos mujer/ingresos } \\
\text { totales }\end{array}$ & $0,41 \quad 0,17$ & & $0,37 \quad 0,18$ & & $0,44 \quad 0,10$ & & \\
\hline $\begin{array}{l}\text { Distribución ingresos mujeres según mediana } \\
\text { ingresos }\end{array}$ & \multicolumn{7}{|c|}{ Porcentajes } \\
\hline hombres & & & & & & & \\
\hline menos del $25 \%$ & 45,7 & & 57,9 & & 54,4 & & \\
\hline $25-50 \%$ & 26,6 & & 16,8 & & 23,2 & & \\
\hline $51-75 \%$ & 15,0 & & 15,6 & & 11,5 & & \\
\hline más del $75 \%$ & 12,7 & & 9,6 & & 10,9 & & \\
\hline \multicolumn{8}{|l|}{$\begin{array}{l}\text { Ingresos mujeres en relación a los ingresos } \\
\text { totales hogar }\end{array}$} \\
\hline menos $20 \%$ & 12,3 & & 20,2 & & 0,7 & & \\
\hline $20-39$ & 31,8 & & 36,4 & & 35,5 & & \\
\hline $40-59$ & 42,0 & & 34,4 & & 57,4 & & \\
\hline $60-80$ & 12,0 & & 7,7 & & 6,5 & & \\
\hline más $80 \%$ & 1,9 & & 1,3 & & - & & \\
\hline \multicolumn{8}{|l|}{ Aporte de ingresos en el hogar } \\
\hline Mujer>Hombre & 26,7 & & 19,7 & & 12,1 & & \\
\hline Mujer=Hombre & 9,1 & & 4,7 & & 34,7 & & \\
\hline Mujer $<$ Hombre & 64,3 & & 75,6 & & 53,2 & & \\
\hline \multicolumn{8}{|l|}{ Tipo de hogar } \\
\hline pareja sola & 17,4 & & 20,5 & & 21,0 & & \\
\hline pareja hijo menor 0-4 & 24,0 & & 20,8 & & 24,3 & & \\
\hline pareja hijo menor 5-9 & 20,2 & & 17,7 & & 16,8 & & \\
\hline pareja hijo menor 10-19 & 26,7 & & 24,2 & & 24,9 & & \\
\hline pareja hijos más 19 & 10,8 & & 15,3 & & 12,9 & & \\
\hline pareja con otros mayor 15 & 1,0 & & 1,6 & & 0,0 & & \\
\hline \multicolumn{8}{|l|}{ Presencia de menores en el hogar } \\
\hline Menores de 4 años & 29,4 & & 26,4 & & 24,3 & & \\
\hline Menores de 4 a 9 años & 30,1 & & 28,2 & & 23,7 & & \\
\hline Menores de 10 a 14 años & 31,9 & & 25,6 & & 23,3 & & \\
\hline Menores de 15 a 19 años & 28,8 & & 24,5 & & 20,9 & & \\
\hline Servicio doméstico en el hogar & 8,8 & & 12,1 & & 16,4 & & \\
\hline
\end{tabular}

Muestra: hogares formados por parejas, ocupadas en el mercado de trabajo y que reciben un salario.

(1) Tiempo social: media y desviación estándar de horas diarias.

Fuente: elaboración propia a partir de la Encuesta Empleo del Tiempo de España, INE (2010); la Encuesta de Trabajo no Remunerado de Argentina, INDEC (2013); y la Encuesta Usos del Tiempo de Chile, INE (2015). 
La subpoblación está formada por los hogares formados por parejas de mujer y hombre, ambos ocupados en el mercado laboral y que reciben salario, con el objeto de examinar con mayor profundidad una de las situaciones de mayor incidencia en la interrelación entre el trabajo remunerado y el trabajo doméstico y de cuidados, siendo además aquellos hogares donde se esperarían unas relaciones más igualitarias en la distribución de los trabajos (Ajenjo y García, 2011; Daly, 2011; Gálvez et al., 2010; Kan et al., 2011; Sayer, 2010; González y Jurado, 2009).

La muestra final, de hogares de parejas de doble ingreso, es de 5.730 hogares en Argentina, 1.671 en Chile y 1.771 en España. La muestra argentina es mucho mayor al formar parte de la operación estadística regular de la Encuesta Permanente de Hogares.

\subsection{Análisis}

Para identificar las variables asociadas a la brecha en la distribución del trabajo doméstico y de cuidados realizado en el hogar, se estima un modelo de regresión (mínimos cuadrados ponderados), para cada uno de los países analizados, donde la variable dependiente es el porcentaje de trabajo doméstico y de cuidados que realiza la persona principal femenina del hogar sobre el total que realizan las dos personas de la pareja. Las variables independientes son los factores que pueden influir en esta distribución. Entre estas se incorporan variables del hogar y de las necesidades de cuidados (tipo de hogar según la presencia y edad de menores, número de menores y servicio doméstico) y variables que dan cuenta de los recursos relativos tanto de las mujeres como de los hombres: edad, nivel educativo, categoría socioprofesional y aporte de los ingresos de la mujer en relación con los ingresos del hombre. Por último, también se ha considerado aquellos factores relacionados con la disponibilidad de tiempo: el tiempo dedicado por ambas personas al trabajo remunerado y el tiempo de carga total de trabajo doméstico y de cuidados que realizan en el hogar las dos personas principales.

En los tres modelos de regresión se utilizan tanto variables cuantitativas como cualitativas, transformadas en variables dicotómicas. En la tabla 3 se presenta la descripción de estas variables, sus categorías y la muestra utilizada (hogares de parejas, con o sin hijos/as u otras personas a su cargo, ocupadas en el mercado laboral y de doble ingreso).

Teniendo en cuenta las aproximaciones teóricas presentadas anteriormente, las principales hipótesis que se plantean son:

(H1) Los recursos relativos de las mujeres tendrán un papel relevante en dar cuenta de la proporción de trabajo doméstico y de cuidados que se realiza: trabajar a tiempo completo, tener un nivel educativo alto, ocupaciones con alto prestigio y aportar más recursos económicos que la pareja reducirán la proporción del trabajo realizado por las mujeres.

(H2) Las variables de los recursos relativos de los hombres tendrán un menor efecto, si no inexistente, lo que nos permitirá comprobar la relevancia 
de la construcción del género para dar cuenta del reparto del trabajo doméstico y de cuidados.

(H3) La presencia de menores en el hogar tendrá un efecto importante en la brecha de género: aumentará la desigualdad entre los dos miembros de la pareja, y por tanto aumentará la carga de trabajo doméstico y de cuidados que asumen las mujeres.

(H4) En aquellos países donde persisten con más fuerza actitudes y opiniones favorables a la adscripción de mujeres y hombres a los trabajos según las relaciones de género, se constatará una mayor brecha de género, así como efectos distintos y con magnitudes distintas de las variables independientes, debido a la relevancia de la visualización de los roles de género.

(H5) Por su parte, en aquellos países donde los valores de género son más igualitarios, y por tanto más igualitaria la distribución del trabajo no remunerado del hogar, menores serán los efectos de las variables independientes.

\section{Resultados}

Un análisis preliminar de los resultados sobre la desigualdad de dedicación en los tiempos de trabajos en las parejas de doble ingreso, tabla 3 , indica que en los tres países la carga global de trabajo de las mujeres es superior a la de los hombres, aproximadamente 1 hora diaria en Argentina y Chile y 40 minutos en España. Esta mayor carga global del trabajo de las mujeres se explica por la menor dedicación masculina a los trabajos domésticos y de cuidados: del total realizado por las dos personas principales, aproximadamente el $70 \%$ es ejecutado por las parejas femeninas, y llega al $76 \%$ en el caso de Argentina. Lo que significa que las mujeres hacen un poco más del doble de trabajo doméstico y de cuidados que los hombres. Los datos muestran que las mujeres se han incorporado al mercado laboral (realizan el $45 \%$ del total de trabajo remunerado de las dos personas principales) sin una redistribución y corresponsabilidad del trabajo doméstico y de cuidados del hogar. La brecha de género es más acentuada en el caso de las mujeres argentinas, donde la ratio de desigualdad del trabajo doméstico, y de carga global del trabajo, es la más alta de los tres países $(2,57)$, y es relevante la desigualdad en el trabajo de cuidados si se compara con Chile y España. Es posible que este resultado pueda deberse a que en la encuesta argentina no se recogía la información del tiempo si como mínimo no se dedicaba una hora diaria, lo que afectó la declaración del trabajo de cuidados de los hombres. En todo caso, lo que sí muestra es su baja dedicación con relación a las mujeres, siendo siempre residual, y la brecha de género en este trabajo.

La primera idea que se destaca de los resultados de los modelos explicativos, tabla 4, es que la capacidad explicativa de las variables individuales, que fundamentalmente están relacionadas con los recursos relativos de las mujeres y de los hombres, tiene un poder explicativo bajo: el 14,6\% i el 16,5\% en Argentina y España; y un porcentaje aún más bajo, del 8,9\%, en el caso de Chile. Ello confirma que los recursos relativos de mujeres y hombres, y su incidencia en 
la capacidad de negociación, tienen un efecto limitado en la explicación de la distribución del trabajo no remunerado dentro del hogar.

De forma concreta, la comparación de la magnitud y dirección de los efectos de las variables consideradas en los modelos de los distintos países revela ciertos aspectos de interés.

En primer lugar, podemos observar que la jerarquía y la magnitud del factor más relevante en explicar la brecha en la distribución del trabajo doméstico y de cuidados en parejas de doble ingreso son distintas según el país. En Argentina, son las horas totales de trabajo doméstico y de cuidados realizadas por las personas principales en el hogar las que tienen mayor impacto en la brecha de género: cuanto más horas de dedicación total, más disminuye la desigualdad en el reparto. En este caso, tal como muestran los datos, la desigualdad en las parejas de doble ingreso argentinas disminuye (en el 1\%) en aquellos hogares donde se realiza mayor trabajo doméstico y de cuidados total. Este efecto visibiliza que la mayor dedicación de los hombres argentinos al trabajo doméstico y de cuidados - y por tanto la disminución en la brecha de género- se produce en hogares donde existen mayores necesidades de cuidados a satisfacer y donde las mujeres no pueden asumir mayor carga (teniendo en cuenta que las mujeres argentinas son las que más tiempo dedican y donde la brecha de género es mayor).

En cambio, en Chile y en España, la variable con un mayor efecto tiene que ver con el varón y su dedicación al trabajo remunerado: cuanto más horas en el mercado, más desigualdad en el reparto del trabajo no remunerado del hogar. Así pues, se constata en estos dos países la relevancia de la disponibilidad de tiempo masculino, en la dedicación al trabajo remunerado.

En España, junto al tiempo masculino en el mercado, también la edad tiene un efecto similar en importancia: a menor edad, menor desigualdad. Ello muestra la tendencia del cambio generacional en las actitudes y relaciones de gmestico y de cuidados.abajo dhogares donde existen mayores necesidades de cuidados a satisfacer y donde las mujeres no pueden asénero, en las parejas de doble ingreso, que se ha dado en España; y por tanto, su mayor capacidad explicativa que el resto de las variables consideradas.

Este primer resultado, centrado en el factor con mayor capacidad explicativa de la desigualdad en el reparto del trabajo no remunerado realizado desde los hogares, nos permite señalar la relevancia del tiempo total dedicado en el hogar para satisfacer las necesidades de bienestar y de cuidados (caso argentino) — a mayor necesidad, menor desigualdad —, y de la disponibilidad del tiempo masculino (en Chile y España) - a mayor dedicación al trabajo remunerado por parte de los hombres, mayor desigualdad en el reparto del trabajo no remunerado.

La baja capacidad explicativa de las variables individuales, el efecto y magnitud distinta observada en los tres países, junto al cambio generacional que se observa en España por parte de los hombres, muestran la relevancia de las ideologías o actitudes de género en dar cuenta de la brecha de género en la realización del trabajo doméstico y de cuidados. 
Para facilitar la lectura de los resultados y comprobar las hipótesis propuestas, se organizará la exposición por bloques temáticos.

\subsection{Los recursos relativos: nivel educativo, categoría socioprofesional e ingresos}

Los resultados muestran que uno de los factores más relevantes es el recurso relativo de los ingresos de las mujeres por su ingreso para explicar la desigualdad en la distribución del trabajo doméstico y de cuidados entre las dos personas principales del hogar en parejas de doble ingreso. En los tres países analizados, un mayor ingreso femenino supone reducir la brecha de desigualdad. Este efecto se observa también si se fija la atención no solo en el ingreso cuantitativo, sino también en el aporte de ingresos de la mujer con relación a su compañero masculino. Tomando como referencia los hogares con igualdad salarial, cuando la mujer aporta ingresos superiores (porcentaje que en ningún país supera el $26 \%$ de los hogares), se reduce en 2 y 3 puntos porcentuales la desigualdad entre mujeres y hombres en España y Chile respectivamente.

Este resultado incide en el papel relevante de los ingresos de las mujeres y su mayor capacidad de negociación, aunque con un efecto pequeño, en la reducción de la brecha de género en el trabajo no remunerado realizado en los hogares.

En Argentina, se constata que los hogares más igualitarios en la distribución del trabajo doméstico y de cuidados son aquellos donde hay mayor igualdad salarial: la brecha de género aumenta en aquellos hogares donde hay desigualdad salarial (aunque sea la mujer la que tenga mayores salarios). Esta situación, contraria a lo que establece la perspectiva teórica de los recursos relativos, puede mostrar la menor capacidad de negociación de las mujeres argentinas y la fuerza e influencia de las normas y los valores de género en la distribución del trabajo del hogar. En contextos menos igualitarios en ideologías de género, aquellas mujeres que las trasgreden por su capacidad de ingresos en el mercado refuerzan su papel de género femenino en el interior del hogar, y por tanto asumen mayor trabajo no remunerado, como una forma de compensar su transgresión a los valores sociales y culturales de género.

Con relación al nivel educativo, los resultados obtenidos reafirman los efectos ambiguos en la reducción de la desigualdad de género en el interior del hogar, debido a su interrelación con los factores de la ideología de género.

Concretamente, un mayor nivel educativo de las mujeres no tiene ningún efecto en la reducción de la desigualdad: solo en Argentina se constata que los niveles educativos femeninos inferiores a los universitarios tienen un efecto amplificador de las desigualdades. No ocurre lo mismo en Chile y en España, donde el nivel educativo de las mujeres no supone cambios significativos en la brecha estudiada.

En cambio, el efecto del nivel educativo de los hombres es mucho más claro y homogéneo en los tres países: los niveles educativos básicos y medios de los hombres, con relación a los niveles universitarios, aumentan las desigualdades en un reparto igualitario. 
Tabla 4. Regresión Mínimos Cuadrados Ordinarios (OLS) de la distribución del trabajo domestico y de cuidados de las personas principales femenina y masculina del hogar (método Stepwise), Argentina, Chile y España

\begin{tabular}{|c|c|c|c|c|c|c|c|c|c|c|}
\hline & \multicolumn{3}{|c|}{ Argentina } & \multicolumn{4}{|c|}{ Chile } & \multicolumn{3}{|c|}{ España } \\
\hline & \multicolumn{2}{|c|}{$\begin{array}{c}\text { Coef. no } \\
\text { estandarizado }\end{array}$} & \multirow{2}{*}{$\begin{array}{c}\text { Coef. } \\
\text { Estandarizado } \\
\text { Beta }\end{array}$} & \multicolumn{3}{|c|}{$\begin{array}{c}\text { Coef. no } \\
\text { estandarizado }\end{array}$} & \multirow{2}{*}{$\begin{array}{c}\text { Coef. } \\
\text { estandarizado } \\
\text { Beta }\end{array}$} & \multicolumn{2}{|c|}{$\begin{array}{c}\text { Coef. no } \\
\text { estandarizado }\end{array}$} & \multirow{2}{*}{$\begin{array}{c}\text { Coef. } \\
\text { estandarizado } \\
\text { Beta }\end{array}$} \\
\hline & B & $\begin{array}{c}\text { Error } \\
\text { estándar }\end{array}$ & & B & & $\begin{array}{c}\text { Error } \\
\text { estándar }\end{array}$ & & B & $\begin{array}{c}\text { Error } \\
\text { estándar }\end{array}$ & \\
\hline (Constante) & $89,287^{\star \star \star}$ & 1,949 & & 79,773 & *** & 8,304 & & 86,124 *** & 8,737 & \\
\hline $\begin{array}{l}\text { Horas totales de } \\
\text { trabajo doméstico } \\
\text { y de cuidados del } \\
\text { hogar }\end{array}$ & $-1,007^{\star \star \star}$ & 0,024 & $-0,282$ & $-0,203$ & & 0,095 & $-0,053$ & $-0,840^{\star \star \star *}$ & 0,122 & $-0,126$ \\
\hline Ingreso (log.) ppf & $-2,457^{\star \star \star}$ & 0,208 & $-0,093$ & $-1,618$ & $\star \star \star \star ~$ & 0,639 & $-0,079$ & 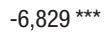 & 1,229 & $-0,127$ \\
\hline Ingreso (log.) ppm & (2) & & $0,009^{(1)}$ & & & (2) & & (2) & & $-0,022^{(1)}$ \\
\hline $\begin{array}{l}\text { Horas trabajo } \\
\text { remunerado ppf }\end{array}$ & 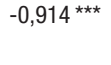 & 0,046 & $-0,136$ & $-0,586$ & 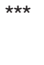 & 0,167 & $-0,098$ & $-1,525^{\star \star \star}$ & 0,213 & $-0,130$ \\
\hline $\begin{array}{l}\text { Horas trabajo } \\
\text { remunerado ppm }\end{array}$ & $0,941^{\star \star \star \star}$ & 0,043 & 0,136 & 1,071 & 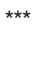 & 0,159 & 0,164 & 2,099 *** & 0,222 & 0,160 \\
\hline Edad ppf & $0,304^{*}$ & 0,194 & 0,016 & & & 0,048 & $-0,077$ & & & $0,040^{(1)}$ \\
\hline Edad ppm & 1,250 *** & 0,218 & 0,061 & 0,208 & $\star \star$ & 0,093 & 0,116 & 0,450 *** & 0,059 & 0,156 \\
\hline \multicolumn{11}{|l|}{$\begin{array}{l}\text { [Nivel educativo } \\
\text { ppf alto] }\end{array}$} \\
\hline $\begin{array}{l}\text { Nivel educativo } \\
\text { ppf básico }\end{array}$ & $2,252^{\star \star \star *}$ & 0,487 & 0,040 & & (2) & & $0,014^{(1)}$ & (2) & & $-0,023^{(1)}$ \\
\hline $\begin{array}{l}\text { Nivel educativo } \\
\text { ppf medio }\end{array}$ & 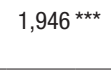 & 0,341 & 0,042 & & (2) & & $-0,006^{(1)}$ & (2) & & $-0,005^{(1)}$ \\
\hline \multicolumn{11}{|l|}{$\begin{array}{l}\text { [Nivel educativo } \\
\text { ppm alto] }\end{array}$} \\
\hline $\begin{array}{l}\text { Nivel educativo } \\
\text { ppm básico }\end{array}$ & $1,835^{\star \star \star *}$ & 0,367 & 0,036 & 4,936 & $\star \star \star \star ~$ & 1,590 & 0,090 & 5,584 *** & 1,549 & 0,081 \\
\hline $\begin{array}{l}\text { Nivel educativo } \\
\text { ppm medio }\end{array}$ & (2) & & $0,004^{(1)}$ & 5,137 & $\star \star \star \star ~$ & 1,188 & 0,123 & $5,290^{\star \star \star}$ & 1,161 & 0,111 \\
\hline CSP ppf 1 & $(2)$ & & $-0,005^{(1)}$ & & (2) & & $-0,008^{(1)}$ & $-2,123^{\star \star}$ & 0,976 & $-0,040$ \\
\hline CSP ppf 2 & (2) & & $0,000^{(1)}$ & & (2) & & $-0,009^{(1)}$ & (2) & & $-0,024^{(1)}$ \\
\hline CSP ppf 3 & $-1,856^{* \star \star}$ & 0,343 & $-0,039$ & & (2) & & $-0,005^{(1)}$ & (2) & & $0,006^{(1)}$ \\
\hline CSP ppf 4 & (2) & & $0,008^{(1)}$ & & (2) & & $-0,005^{(1)}$ & (2) & & $-0,005^{(1)}$ \\
\hline \multicolumn{11}{|l|}{$\begin{array}{l}\text { [CSP ppf no } \\
\text { cualific./no clas.] }\end{array}$} \\
\hline CSP ppm 1 & $-2,670^{\star \star \star \star}$ & 0,736 & $-0,044$ & & (2) & & $-0,019$ & 5,649 *** & 1,164 & 0,108 \\
\hline CSP ppm 2 & $-6,170^{\star \star \star}$ & 0,739 & $-0,096$ & & (2) & & $0,034^{(1)}$ & (2) & & $0,005^{(1)}$ \\
\hline CSP ppm 3 & $-4,042^{\star \star \star}$ & 0,726 & $-0,062$ & $-4,010$ & $\star \star \star$ & 1,467 & $-0,066$ & $(2)$ & & $-0,021^{(1)}$ \\
\hline CSP ppm 4 & $-2,889$ *** & 0,655 & $-0,065$ & & (2) & & $0,015^{(1)}$ & 4,100 *** & 0,929 & 0,080 \\
\hline \multicolumn{11}{|l|}{$\begin{array}{l}\text { [CSP ppm no } \\
\text { cualific./no clas.] }\end{array}$} \\
\hline $\begin{array}{l}\text { Hogar pareja } \\
\text { hijo 0-4 }\end{array}$ & $8,192^{\text {*** }}$ & 0,574 & 0,158 & & (2) & & $0,035^{(1)}$ & $3,802^{\text {*** }}$ & 1,275 & 0,071 \\
\hline $\begin{array}{l}\text { Hogar pareja } \\
\text { menor 5-9 }\end{array}$ & 8,254 *** & 0,552 & 0,150 & & (2) & & $-0,005^{(1)}$ & $5,917^{\star \star \star \star}$ & 1,286 & 0,094 \\
\hline $\begin{array}{l}\text { Hogar pareja } \\
\text { menor } 10-19\end{array}$ & $6,686^{\star \star \star \star}$ & 0,480 & 0,134 & & (2) & & $0,017^{(1)}$ & $4,802^{\star \star \star *}$ & 1,179 & 0,087 \\
\hline
\end{tabular}

(continúa) 
Tabla 4. Regresión Mínimos Cuadrados Ordinarios (OLS) de la distribución del trabajo domestico y de cuidados de las personas principales femenina y masculina del hogar (método Stepwise), Argentina, Chile y España (continuación)

\begin{tabular}{|c|c|c|c|c|c|c|c|c|c|c|}
\hline & \multicolumn{3}{|c|}{ Argentina } & \multicolumn{3}{|c|}{ Chile } & \multicolumn{4}{|c|}{ España } \\
\hline & \multicolumn{2}{|c|}{$\begin{array}{c}\text { Coef. no } \\
\text { estandarizado }\end{array}$} & \multirow{2}{*}{$\begin{array}{c}\text { Coef. } \\
\text { Estandarizado } \\
\text { Beta }\end{array}$} & \multicolumn{2}{|c|}{$\begin{array}{c}\text { Coef. no } \\
\text { estandarizado }\end{array}$} & \multirow{2}{*}{$\begin{array}{c}\begin{array}{c}\text { Coef. } \\
\text { estandarizado }\end{array} \\
\text { Beta }\end{array}$} & \multicolumn{3}{|c|}{$\begin{array}{c}\text { Coef. no } \\
\text { estandarizado }\end{array}$} & \multirow{2}{*}{$\begin{array}{c}\begin{array}{c}\text { Coef. } \\
\text { estandarizado }\end{array} \\
\text { Beta }\end{array}$} \\
\hline & B & $\begin{array}{c}\text { Error } \\
\text { estándar }\end{array}$ & & B & $\begin{array}{c}\text { Error } \\
\text { estándar }\end{array}$ & & B & & $\begin{array}{c}\text { Error } \\
\text { estándar }\end{array}$ & \\
\hline $\begin{array}{l}\text { Hogar pareja hijos } \\
\text { + de } 19\end{array}$ & 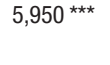 & 0,547 & 0,083 & (2) & & $0,007^{(1)}$ & 7,159 & $\star \star \star \star ~$ & 1,560 & 0,095 \\
\hline $\begin{array}{l}\text { Hogar pareja con } \\
\text { otros + de } 15\end{array}$ & 3,279 ** & 1,370 & 0,015 & (2) & & $-0,016^{(1)}$ & & (2) & & $-0,005^{(1)}$ \\
\hline \multicolumn{11}{|l|}{ [Hogar pareja sola] } \\
\hline \multicolumn{11}{|l|}{$\begin{array}{l}{[\text { Aporte ingresos }} \\
\text { hogar } \mathrm{M}=\mathrm{V}]\end{array}$} \\
\hline $\begin{array}{l}\text { Aporte ingresos } \\
\text { hogar } M>V\end{array}$ & $2,669 * \star \star$ & 0,515 & 0,053 & $-3,150$ *夫 & 1,353 & $-0,060$ & $-2,204$ & & 1,313 & $-0,030$ \\
\hline $\begin{array}{l}\text { Aporte ingresos } \\
\text { hogar } M<V\end{array}$ & 3,435 *** & 0,483 & 0,074 & (2) & & $-0,011^{(1)}$ & & (2) & & $-0,003^{(1)}$ \\
\hline $\begin{array}{l}\text { Núm. menores } \\
\text { hogar }\end{array}$ & $0,692^{\star \star \star *}$ & 0,140 & 0,042 & (2) & & $0,026^{(1)}$ & & (2) & & 0,027 \\
\hline \multicolumn{11}{|l|}{$\begin{array}{l}\text { [No servicio } \\
\text { doméstico hogar] }\end{array}$} \\
\hline $\begin{array}{l}\text { Sí servicio } \\
\text { doméstico hogar }\end{array}$ & $2,777^{\star \star \star}$ & 0,501 & 0,036 & (2) & & $-0,019^{(1)}$ & 1,696 & * & 1,145 & 0,026 \\
\hline F Statistic (df) & \multicolumn{3}{|c|}{$180,689(23)$} & \multicolumn{3}{|c|}{$17,05(10)$} & \multicolumn{4}{|c|}{$41,006(17)$} \\
\hline Adjusted $\mathrm{R}^{2}$ & \multicolumn{3}{|c|}{0,146} & \multicolumn{3}{|c|}{0,089} & \multicolumn{4}{|c|}{0,165} \\
\hline
\end{tabular}

${ }^{*} \mathrm{P}$-value $<0.10 ;{ }^{* *} \mathrm{P}$-value $<0.05 ;{ }^{* *} \mathrm{P}$-value $<0.01$

(1) En las variables no estadísticamente significativas, el coeficiente de regresión estandarizado es el «beta dentro».

(2) Variables no estadísticamente significativas con un valor $-\mathrm{P}>0.10$. Estas variables se excluyen del modelo.

Muestra: hogares formados por parejas, ocupados en el mercado de trabajo y que reciben un salario por su ocupación. ppm = persona principal masculina del hogar; $\mathrm{ppf}=$ persona principal femenina del hogar

[categoría de la variable omitida]

Fuente: elaboración propia a partir de la Encuesta Empleo del Tiempo de España, INE (2010); la Encuesta de Trabajo no Remunerado de Argentina, INDEC (2013); y la Encuesta Usos del Tiempo de Chile, INE (2015).

Así pues, el recurso relativo significativo en explicar la desigualdad en un reparto equitativo en el trabajo doméstico y de cuidados en las parejas de doble ingreso es el recurso educativo bajo de los hombres, que tiene un efecto amplificador de las desigualdades. Solo en el caso argentino, el nivel educativo alto de la mujer tiene un efecto reductor de la desigualdad dentro del hogar.

Si centramos la mirada en las categorías socioprofesionales, las características particulares de los mercados de trabajo y la plasticidad de la significación del prestigio ocupacional inciden en la divergencia de resultados según el país. En Chile la categoría socioprofesional de las mujeres no otorga ningún efecto significativo en la reducción de la brecha de género. En España y Argentina, por su parte, se observa menor desigualdad en la categoría femenina más alta (poder 
legislativo y judicial; directivas y técnicas altas y científicas) y en la categoría de las trabajadoras de los servicios y el comercio, respectivamente. En este sentido, se entrevé la consecuencia de la visualización de los roles y las ideologías de género en la distribución del trabajo doméstico y de cuidados en mujeres y hombres.

Por su parte, tal como se observa en los niveles educativos, el efecto de la categoría socioprofesional de los hombres tiene un mayor poder explicativo, particularmente en Argentina, donde los hombres de todas las categorías socioprofesionales, en comparación con los hombres no cualificados, tienen como efecto una disminución de la desigualdad en el reparto; de forma especial en las categorías de técnicos medios, empleados de oficina y los trabajadores de los servicios y el comercio. Esta misma última categoría (los trabajadores de los servicios y el comercio) es la única que tiene un efecto de disminución de la desigualdad en Chile.

Las categorías socioprofesionales de los hombres españoles solo muestran un efecto significativo, de aumento de la desigualdad, en el caso de los directivos y técnicos altos y los trabajadores cualificados.

Estos resultados respaldan que las diferencias internas entre los hombres, su estratificación socioeconómica según sus recursos relativos, son más importantes que entre las mujeres para explicar la brecha de género en la división sexual del trabajo en el interior del hogar.

Como conclusión de este apartado, se puede señalar que los recursos relativos de mujeres y hombres y su impacto en comportamientos más igualitarios en la distribución del trabajo doméstico y de cuidados tienen una especial relevancia en el caso de los salarios y aportes económicos de las mujeres en el hogar. Los recursos relativos de las mujeres, y su capacidad de negociación, vía el nivel educativo y las categorías socioprofesionales tienen un menor papel, acaso inexistente, y están fuertemente imbricados con las ideologías o actitudes de género. Mostrando que la estratificación socioeconómica de las mujeres está atravesada por las relaciones de género.

En cambio, los recursos relativos de los hombres, su nivel educativo y categoría socioprofesional son más consistentes y con una mayor magnitud, especialmente en el caso argentino. Ello puede ser reflejo de las ideologías y los valores de género masculinos más desigualitarios, y de una mayor incidencia de la estratificación socioeconómica masculina en la brecha de género: sus posiciones relativas tienen mayor impacto en su dedicación al trabajo doméstico y de cuidados, y en consecuencia en la distribución del tiempo en el hogar.

\subsection{La disponibilidad de tiempo: las horas de trabajo remunerado}

La perspectiva teórica de la disponibilidad de tiempo señala que el aumento de las horas de dedicación al trabajo remunerado de las mujeres inferirá una disminución de su tiempo de dedicación al trabajo doméstico y de cuidados y un aumento de la dedicación de los hombres. Por tanto, es esperable una disminución de la brecha de la desigualdad en el reparto del trabajo doméstico y de cuidados en las parejas de doble ingreso. 
Los resultados confirman estos presupuestos: a mayor dedicación al trabajo de mercado de las mujeres, disminuye la desigualdad en las parejas de doble ingreso, con un efecto mayor en España (con una reducción de 1,5 puntos porcentuales) que en Chile, donde disminuye 0,5 puntos porcentual.

Lo que es destacable es el efecto observado cuando es el hombre quien aumenta su dedicación horaria al trabajo de mercado. En primer lugar, disminuye su dedicación al trabajo doméstico y de cuidados, aumentando la brecha de género. Ante esta disminución la mujer, ocupada también en el mercado, o bien asume la parte que deja de realizar en el hogar su compañero, o bien externaliza parte de este trabajo. Con las consecuencias del alargamiento, e intensidad, del tiempo de la carga global del trabajo de las mujeres o con una reducción del trabajo de mercado, lo que aumenta la brecha de género y afecta la participación laboral.

Igualmente relevante es que el efecto del aumento de la dedicación masculina al trabajo de mercado tiene un efecto amplificador de la desigualdad mucho mayor que el efecto reductor observado cuando es la mujer la que aumenta su dedicación al mercado. Aumentar una hora semanal la dedicación al empleo impacta en la brecha de género: $+2,09$ si quien aumenta es el hombre frente a $-1,5$ si aumenta la mujer en España; $y+1,07$ del hombre frente a $-0,58$ de la mujer en Chile. En Argentina, el efecto de aumento $(+0,94)$ y el de reducción $(-0$ 91) de la brecha de género es el mismo cuando hombres y mujeres dedican una hora más al trabajo de mercado.

Estos resultados son un indicador de que la mayor dedicación horaria masculina al trabajo de mercado no solo supone una reducción de su trabajo doméstico y de cuidados, sino que, como consecuencia, las mujeres tienen que reducir su dedicación al mercado para asumir la disminución del trabajo del hogar de los hombres, o bien externalizar parte de él. Por tanto, la disponibilidad de tiempo y su efecto en la brecha de género en la carga de trabajo doméstico y de cuidados, y en la carga global de trabajo, se adecuan a la estrategia masculina, pero no a la femenina, que responde más a las necesidades de cuidados del hogar entreveradas por relaciones e ideología de género.

\subsection{Ciclo vital y necesidades de cuidados: edad, estructura familiar y servicio doméstico remunerado}

La necesidad de cuidados del hogar es uno de los factores fundamentales cuando se da cuenta de la distribución, y la brecha de género, del trabajo doméstico y de cuidados. En parejas de doble ingreso, la presencia de menores ${ }^{6}$ en el hogar es una de las situaciones más críticas, que supone un aumento de las necesi-

6. También la presencia de personas ancianas y adultas dependientes inciden fuertemente en la dedicación al trabajo de cuidados de mujeres y hombres. En este estudio no se ha tenido en cuenta dadas las dificultades de medición en las tres encuestas utilizadas. Tampoco se disponía de información homogénea sobre el cuidado asumido por instituciones públicas y por el mercado. 
dades de cuidados del hogar y el establecimiento de estrategias familiares para satisfacerlas. De forma especial, en aquellas parejas más jóvenes (que tienen hijos/as menores de 4 y de 5 a 9 años).

Los resultados que se observan, tomando como referencia a las parejas de hogares sin otros miembros en el hogar, señalan que Chile mantiene una pauta totalmente distinta a España y Argentina: la presencia de menores en el hogar, sea cual sea su edad, no tiene ningún efecto significativo en la brecha de distribución de tiempos domésticos y de cuidados de mujeres y hombres, y no se modifica el patrón de desigualdad en la dedicación. Esto es, la presencia de menores aumenta el trabajo doméstico y de cuidados, pero no modifica en ningún caso la pauta de distribución desigualitaria entre las dos personas principales de la pareja. Ambas, mujeres y hombres, aumentan su dedicación, por el aumento de trabajo de cuidados, pero no modifican las relaciones de género en la corresponsabilidad por la presencia de menores. Así por ejemplo, en parejas de doble ingreso sin menores a su cargo, las mujeres chilenas dedican una media de 4,11 horas diarias al trabajo doméstico y de cuidados frente a 2,14 horas de sus compañeros masculinos. Cuando las parejas cuentan con menores de 4 años a su cargo, ellas aumentan su dedicación a 9,41 horas y ellos a 4,54 (se mantiene, aumentando levemente, la desigualdad).

Estos resultados muestran la inmutabilidad de las relaciones de género desiguales en la distribución de los trabajos en el interior de los hogares con la presencia de niños/as pequeños/as y lo que supone de aumento del trabajo no remunerado realizado desde el hogar.

Los resultados en el caso argentino y español constatan situaciones mucho más desigualitarias. La presencia de menores, menos de 4 años y hasta 9 años, tienen como efecto el aumento de las desigualdades por género: son las situaciones en que las mujeres asumen mayor carga de trabajo doméstico y de cuidados en relación a sus compañeros masculinos. Situaciones en que el aumento de las desigualdades es mayor comparado con el resto de variables. Lo que es destacable es que la desigualdad se acrecienta en el caso de que los hijos/as tengan edades entre 5 a 9 años: la brecha de género aumenta en 8,25 y 5,91 puntos porcentuales en Argentina y España, respectivamente. La situación es especialmente gravosa para las mujeres argentinas. Seguramente sería necesario disponer de información del papel de los servicios públicos y de mercado que las familias utilizan, pero más allá de ello, la brecha de género en el trabajo de cuidados que se realiza en el hogar es contundente en estos países.

La edad de la pareja nos puede aportar información sobre el comportamiento en las parejas de doble ingreso más jóvenes. Hasta qué punto se puede observar un cambio en las relaciones desigualitarias. Los resultados nos permiten afirmar que la edad de los hombres tiene un efecto significativo, manteniendo constante el resto de variables, igual en los tres países analizados: a medida que aumenta la edad de los hombres, aumenta la desigualdad. De forma especial en los hombres argentinos (por cada año mayor, aumenta 1,25 puntos porcentua- 
les la desigualdad). Se confirma entonces la tendencia de cambio generalizado, en los hombres más jóvenes.

Los efectos de la edad de las mujeres muestran la incidencia y el mantenimiento de las relaciones de género. En Chile y España no tiene un efecto significativo, esto es, sea cual sea la edad de la mujer, se mantienen los comportamientos desiguales, lo que produce un efecto de disminución es la edad de los hombres.

En Argentina, la edad de las mujeres tiene el mismo efecto significativo, aunque con un impacto menor que el de los hombres: cuando aumenta la edad, aumenta la desigualdad. En este caso, se constata también un cambio de comportamiento: mayor igualdad en las parejas más jóvenes, tanto por los efectos masculinos como femeninos.

Por otro lado, prestar atención a la carga global de tiempo doméstico y de cuidados realizada por los dos miembros principales nos puede proporcionar elementos de análisis acerca de cómo inciden aquellas situaciones donde se realiza un mayor tiempo de trabajo no remunerado en el hogar. Además permite comprobar si la disminución de la desigualdad se produce en hogares donde se realiza poco trabajo doméstico y de cuidados (sería más fácil compartir cuando se realiza poco trabajo). En este sentido, los efectos de esta variable, consistentes en los tres países, muestran que a mayor tiempo total realizado por las personas principales del hogar, menor es la brecha de dedicación entre mujeres y hombres. Así pues, en aquellos hogares donde es mayor el trabajo no remunerado, manteniéndose el patrón general que la mujer asume mayor carga de trabajo, más disminuye la desigualdad. De forma especial en los hogares argentinos, disminución de 1,00 frente a los chilenos, 0,28 . Resultado que apunta a que la dedicación de los hombres al trabajo no remunerado, siendo baja, aumenta en aquellas situaciones en que la carga de trabajo doméstico y de cuidados se hace insostenible e imposible de asumir por las mujeres.

Por último, cabe analizar el efecto que tiene el servicio doméstico remunerado realizado en el hogar. $\mathrm{Al}$ igual que la presencia de menores, en España y Argentina, disponer de servicio doméstico remunerado en el hogar tiene como efecto un aumento de la desigualdad en la brecha de dedicación: en aquellos hogares donde hay servicio doméstico remunerado, aumenta la desigualdad entre mujeres y hombres: 1,7 y 2,7 puntos porcentuales en España y Argentina respectivamente. Así pues, cuando una parte del trabajo doméstico y de cuidados es realizado por otra persona (mujer mayoritariamente) de forma remunerada en el hogar, esto supone una reducción del trabajo que realizan los hombres, pero no del trabajo asumido por las mujeres. Seguramente, las mujeres reducen una parte del trabajo doméstico y de cuidados, el más rutinario y fácilmente externalizable, pero son ellas las que gestionan, organizan y «están pendientes» de tareas y cuidados. En cambio, para los hombres, más ajenos a estos trabajos de organización y planificación, el servicio doméstico remunerado supone una reducción neta de su aportación en tiempos y dedicación. Reducción mucho mayor en tiempo que la de su compañera femenina, con lo que la brecha de género aumenta. 


\section{Conclusiones}

Retomando las hipótesis establecidas, podemos señalar que, contrariamente a lo establecido, los recursos relativos de las mujeres tienen un menor efecto en la brecha de género del trabajo doméstico y de cuidados que los recursos relativos de los hombres. Tan solo el aporte de ingresos de las mujeres se muestra relevante en la reducción de la brecha de género.

La notable importancia de los recursos relativos de los hombres (su dedicación al trabajo remunerado, su nivel educativo y sus categorías socioprofesionales) señala la incidencia del efecto de la estratificación socioeconómica masculina sobre el reparto del trabajo no remunerado realizado por las personas principales del hogar y en los valores de género de los hombres.

Así pues, los resultados muestran la necesidad de incorporar la ideología de género y los elementos contextuales en la explicación de la brecha de género en el interior de los hogares.

La comparación entre los tres países da cuenta de la relevancia de los contextos sociales y los valores sociales y culturales menos igualitarios.

En Chile y Argentina, persisten con más fuerza valores, normas sociales y culturales androcéntricas y de adscripción de mujeres y hombres a los trabajos según las relaciones de género. Así pues, en Argentina la brecha de género es mayor, al igual que son mayores los efectos de las variables de los recursos relativos y la estratificación socioeconómica, tanto de las mujeres como de los hombres. Las desigualdades en las relaciones sociales de producción se entrecruzan fuertemente con el mantenimiento de las relaciones de desigualdad por género. En consecuencia, las mujeres de clases populares y de hogares más desfavorecidos asumen una mayor desigualdad en el trabajo no remunerado dentro de los hogares.

En Chile, la brecha de género es escasamente explicada por el modelo de características individuales. Son los elementos contextuales y la ideología de género, marcadamente desigualitaria, los factores que subyacen en la división sexual del trabajo. Los valores androcéntricos que perviven en la sociedad chilena se manifiestan de forma contundente en un contexto en que los recursos relativos de las mujeres inciden muy poco en la brecha de género en el interior de los hogares, y son las actitudes y los valores masculinos los que tienen mayor impacto en la dedicación masculina al trabajo no remunerado, y por tanto en los procesos de desigualdad.

España, con una menor brecha de género, se caracteriza, por la relevancia de los recursos relativos y la disponibilidad de tiempo de los hombres en dar cuenta de los cambios en la división más equitativa del trabajo doméstico y de cuidados. Y en las transformaciones en la ideología de género, más favorables a la igualdad, que pueden venir protagonizadas por las generaciones más jóvenes.

En conclusión, se visibiliza que las mujeres mantienen una dedicación al trabajo doméstico y de cuidados en consonancia con las necesidades de cuidados y de bienestar de las personas del hogar. La brecha de género es sensible a ello y muestra que el tiempo femenino es la variable de ajuste. Y por tanto, con una gravosa incidencia en la calidad de vida y el bienestar de las mujeres. 


\section{Referencias bibliográficas}

AgUiRre, Rosario (ed.) (2009). Las bases invisibles del bienestar social. El trabajo no remunerado en Uruguay. Montevideo: Fondo de Desarrollo de la Naciones Unidas para la Mujer.

AgUirRe, Rosario y Ferrari, Fernanda (2014). Las encuestas sobre uso del tiempo y trabajo no remunerado en América Latina y el Caribe. Caminos recorridos y desafíos hacia el futuro. Santiago: CEPAL.

- (2015). «Avances en la medición del uso del tiempo y el trabajo no remunerado en la región». En: BATTHYÁNY, Karina (2015). Los tiempos del bienestar social. Montevideo: Instituto Nacional de las Mujeres. Ministerio de Desarrollo Social.

Aguirre, Rosario; García, Cristina y Carrasco, Cristina (2005). «El tiempo, los tiempos, una vara de desigualdad». Serie Mujer y Desarrollo, 65. Santiago de Chile: CEPAL.

AjEnjo, Marc y García, Joan (2011). «El tiempo productivo, reproductivo y de ocio en las parejas de doble ingreso». Papers, 96 (3), 985-1006. $<$ https://doi.org/10.5565/rev/papers/v96n3.183>.

Albelda, Randy; Duffy, Mignon y Folbre, Nancy (2009). Counting on care work: Human infrastructure in Massachusetts (2009). Center for Social Policy Publications, 33.

Amarante, Verónica y Rossel, Cecilia (2017). «Unfolding patterns of unpaid household work in Latin America». Feminist Economics, 24 (1), 1-34. <https://doi.org/10.1080/13545701.2017.1344776>.

ANTONOPOULOS, Rania (2008). "The unpaid care work-paid work connection». Working Papers Series. Levy Economics Institute. $<$ https://doi.org/10.2139/ssrn.1176661>.

ANTONOPOUlOS, Rania y HiRWAY, Indira (ed.) (2010). Unpaid work and the economy gender, time use and poverty in developing countries. Londres: Palgrave Macmillan. <https://doi.org/10.1057/9780230250550>.

ARriagAdA, Irma (2007). Familias y politicas públicas en América Latina: una historia de desencuentros. Santiago de Chile: CEPAL.

BALCELLS, Laia (2009). "Analyzing the division of household labor within Spanish families». Revista Internacional de Sociología, 67 85-103. <https://doi.org/10.3989/ris.2009.i1.123>.

Bartley, Sharon; Blanton, Priscilla y Gilliard, Jennifer (2005). «Husbands and wives in dual-earner marriages: Decision making, gender role attitudes, division of household labor, and equity». Marriage and Family Review, 37 (4), 69-94. <https://doi.org/10.1300/J002v37n04_05>.

BATTHYÁNY, Karina (ed.) (2015a). Los tiempos del bienestar social. Instituto Nacional de las Mujeres. Montevideo: Ministerio de Desarrollo Social.

- (2015b). Las políticas y el cuidado en América Latina: una mirada a las experiencias regionales. Santiago de Chile: CEPAL.

BernhardT, Eva; NoAcK, Turid y LyngSTad, Torkild Hovde (2008). «Shared housework in Norway and Sweden: Advancing the gender revolution». Journal of European Social Policy (18), 275-288. <https://doi.org/10.1177/0958928708091060>.

BetTiO, Francesca; Corsi, Marcella; D'IPPOLITI, Carlo; LyBeraKi, Antigone; SAMEK, Manuela y Verashchagina, Alina (2012). The impact of the economic crisis on the situation of women and men and on gender equality policies. Luxemburgo: Unión Europea. 
BianCHI, Suzanne y RALEY, Sara (2005). «Time allocation in working families». En: Bianchi, Suzanne; CASPer, Lynne y Berkowitz, Rosalind. Work, family, health, and well-being. Nueva York: Lawrence Erlbaum. <https://doi.org/10.4324/9781410613523>.

Bianchi, Suzanne; Milkie, Melissa; SAYer, Liana y Robinson, John (2000). «Is anyone doing the housework? Trends in the gender division of household labor». Social Forces (79), 191-228. <https://doi.org/10.1093/sf/79.1.191>.

Bittman, Michael; England, Paula; Sayer, Liana; Folbre, Nancy y Matheson, George (2003). "When does gender trump money? Bargaining and time in household work». American Journal of Sociology, 109 (1), 186-214. $<$ https://doi.org/10.1086/378341>.

Blofield, Merike y MARTíneZ, Juliana (2014). «Trabajo, familia y cambios en las políticas públicas en América Latina: equidad, maternalismo y corresponsabilidad». Revista de la CEPAL (114), 107-125. <https://doi.org/10.18356/d81c1957-es>.

BONKE, Jens (2005). «Paid work and unpaid work: Diary information versus questionnaire information». Social Indicators Research, 70, 349-368. <https://doi.org/10.1007/s11205-004-1547-6>.

BRINES, Julie (1994). "Economic dependency, gender, and the division of labor at home». American Journal of Sociology, 100 (3), 652-88. <https://doi.org/10.1086/230577>.

BuDlENDER, Debbie (2010). What do time use studies tell us about unpaid care work? Evidence from seven countries a time use studies and unpaid care work. Nueva York: Routledge.

Calderón, Coral (2013). «Redistribuir el cuidado. El desafío de las políticas». Cuadernos de la CEPAL, 101. Santiago de Chile: ONU

Calero, Analía; Dellavalle, Rocío y Zanino, Carolina. (2015). Uso del tiempo y economía del cuidado. Documento de trabajo no 09. Secretaría de política económica y planificación del desarrollo. Buenos Aires: Subsecretaría Programación Económica.

CAMPAÑA, Juan Carlos; GiménEZ-NADAL, José Ignacio y MolinA, José Alberto (2017). "Gender norms and the gendered distribution of total work in Latin American households». Feminist Economics, 24 (1), 35-62. <https://doi.org/10.1080/13545701.2017.1390320>.

Carrasco, Cristina y Domínguez, Màrius (2011). «Family strategies for meeting care and domestic work needs: Evidence from Spain». Feminist Economics, 17 (4), 159-188. <https://doi.org/10.1177/0961463X14538917>.

- (2014). «Measured time, perceived time: A gender bias». Time and Society, 24 (3), 326-347. Sage Publications. <https://doi.org/10.1177/0961463X14538917>.

CEPAL (2016a). Autonomía de las mujeres e igualdad en la agenda de desarrollo sostenible. Santiago de Chile.

- (2016b). La matriz de la desigualdad social en América Latina. Santiago de Chile.

- (2016c). Panorama de America Latina 2016. Santiago de Chile: Naciones Unidas.

- (2017a). Repositorio de información sobre uso del tiempo de América Latina y el Caribe. Santiago de Chile.

- (2017b). Estrategia de Montevideo para la Implementación de la Agenda Regional de Género en el marco del Desarrollo Sostenible hacia 2030. Santiago de Chile. 
CEPAL/INEGI/INMUJERES/ONU-Mujeres (2016). Clasificación de actividades de uso del tiempo para América Latina y el Caribe. Santiago de Chile.

Coltrane, Scott (2000). «Research on household labor: Modeling and measuring the social embeddedness of routine family work». Journal of Marriage and Family, 62, 1208-1233. <https://doi.org/10.1111/j.1741-3737.2000.01208.x>.

Coltrane, Scott y Michele, Adams (2001). «Men’s family work: Child-centered fathering and the sharing of domestic labor». En: HERTZ, Rosanna y MARSHALL, Nancy. Working families: The transformation of the American home. Berkeley: University of California Press.

COVERMAN, Shelley (1985). «Explaining husbands' participation in domestic labor». Sociological Quarterly, 26 (1), 81-97. <https://doi.org/10.1111/j.1533-8525.1985.tb00217.x>.

Crompton, Rosemary; Brockmann, Michaela y Lyonette, Clare (2005). «Attitudes, women's employment and the domestic division of labour: A cross national analysis in two waves». Work, employment and Society, 19, 213-233. $<$ https://doi.org/10.1177/0950017005053168>.

DALY, Mary (2011). "What adult worker model? A critical look at recent social policy reform in Europe from a gender and family perspective». Social Politics, 18 (1), 1-23. $<$ https://doi.org/10.1093/sp/jxr002>.

DAVIS, Shannon; GREENSTEIN Theodore y GERTEISEN, Marks (2007). «Effects of union type on division of household labor: Do cohabiting men really perform more housework?». Journal of Family Issues, 28 (9), 1246-1272. <https://doi.org/10.1177/0192513X07300968>.

DEMA, Sandra (2006). Una pareja, dos salarios: El dinero y las relaciones de poder en las parejas de doble ingreso. Madrid: Centro de Investigaciones Sociológicas.

DeUTSCH, Francie; LuSsier, Julianne y SERVIS, Laura (1993). "Husbands at home: Predictors of paternal participation in childcare and housework». Journal of Personality and Social Psychology, 65 (6), 1154-1166. <http://dx.doi.org/10.1037/0022-3514.65.6.1154>.

DíAZ, Capitolina; DeMA, Sandra y FinKel, Lucila (2015). «Desigualdades de género en la distribución de recursos económicos en las parejas españolas». Papers, Revista de Sociología, 100 (1), 53-73.

<http://dx.doi.org/10.5565/rev/papers.591 dx.doi.org/10.5565/rev/papers.591>.

DomíngueZ, Marta (2012). «La división del trabajo doméstico en las parejas españolas. Un análisis de uso del tiempo». Revista Internacional de Sociología, 70 (1), 153-179. <https://doi.org/10.3989/ris.2009.08.26>.

DunN, Dana y SKAGGS, Sheryl (2006). «Gender and paid work in industrial nations». En: Saltzman, Janet y Kluwer, Chafetz. Handbook of the sociology of gender (Handbooks of Sociology and Social Research). Boston: Springer. <https://doi.org/10.1007/0-387-36218-5_15>.

Durán, María Ángeles y MilosaVijeVIC, Vivian (2012). Unpaid work, time use surveys and care demand. Forecasting in Latin America. Bilbao: Fundación BBVA.

Espejo, Andrés; Filgueira, Fernando y Rico, María Nieves (2010). Familias latinoamericanas: organización del trabajo no remunerado y de cuidado. Santiago de Chile: CEPAL.

ESQUIVEL, Valeria (2010). «Lessons from the Buenos Aires time-use survey: A methodological assessment». En: ANTONOPOULOS, Raina. Unpaid work and the economy gender, time use and poverty in developing countries. Londres: Palgrave Macmillan. 
- (2011). La economía del cuidado en América Latina: Poniendo a los cuidados en el centro de la agenda. Buenos Aires: PNUD.

Eurostat (2004). How European spend their time. Everyday life of women and men. Luxemburgo: Comision Europea.

- (2008). Harmonised European time use surveys. Guidelines. Methodologies and Working Papers. Luxemburgo: Comision Europea.

Ferrant, Gaëlle; Pesando, Luca, Maria y Nowacka, Keiko (2014). Unpaid care work: The missing link in the analysis of gender gaps in labour outcome. Ginebra: OECD.

FILGUEIRA, Fernando (2007). "Cohesión, riesgo y arquitectura de protección social en América Latina». Serie de Politicas Sociales, 135. Santiago: CEPAL.

FOLBRE, Nancy (2008). Valuing children rethinking the economics of the family. Cambridge, Mass: Harvard University Press.

Fórum Económico Mundial (2017). Global Gender Gap Report, 2017. Ginebra: Fórum Económico Mundial.

Francavilla, Francesca; Giannelli, Gianna Claudia; GrotKowsKa, Gabriel; PiCCOLI, Luca y SOCHA, Mieczyslaw W. (2009). Women and unpaid family care work in the EU. Bruselas: Parlamento Europeo.

FrancaVilla, Francesca; Giannelli, Gianna; MangiaVACCHI, Lucia y PiCCOl, Luca (2013). «Unpaid work in Europe: Gender and country differences». En: BETTIO, Francesca; Plantenga, Janneke y SMith, Mark. Gender and European labour market. Abigdon: Routledge.

FuWA, Makiko (2004). «Macro-level gender inequality and the division of household labor in 22 countries». American Sociological Review, 69, 751-767. <https://doi.org/10.1177/000312240406900601>.

Fuwa, Makiko y CoHEN, Philip N. (2007). «Housework and social policy». Social Science Research, 36 (2), 512-30. <http://dx.doi.org/10.1016/j.ssresearch.2006.04.005>.

Gálvez, Lina; Rodríguez-Modroño, Paula y Domínguez-Serrano, Mónica (2010). «Género, trabajos y usos del tiempo en España dentro del contexto europeo». En: VILLAR, Antonio (ed.). Mujeres y mercado laboral en España: cuatro estudios sobre la discriminación salarial y la segregación laboral. Bilbao: FBBVA.

GARCÍA, Joan (2012). El uso del tiempo en las parejas de doble ingreso. Tesi doctoral, UAB. Bellaterra: Universitat Autònoma de Barcelona.

GEIST, Claudia (2005). "The welfare state and the home: Regime differences in the domestic division of labour». European Sociological Review, 21 (1) 23-41. <https://doi.org/10.1093/esr/jci002>.

Gesis-LeIBNIZ InSTITUTE FOR THE SOCIAL SCIENCES (2017). International Social Survey Programme, ISPP. Gesis Germany. <http://www.issp.org/menu-top/home/>.

Gershuny, Jonathan (2000). Changing. Times: Work and leisure in postindustrial. Society. Oxford: Oxford University Press.

GERSHUNY, Jonathan y Sullivan, Oliver (2003). «Time use, gender, and public policy regimes». Social Politics, 10, 205-28. $<$ https://doi.org/10.1093/sp/jxg012>.

Gómez, Verónica; Arellano, Oriana y Valenzuela, Cristina. (2017). «Negociaciones en familia: género, trabajo y cuidado en Chile». Estudios Feministas, 25 (2), 562. Florianópolis. <http://dx.doi.org/10.1590/1806-9584.2017v25n2p661>. 
GonZÁLEZ-LÓPEZ, María José (2001). «Spouse’s employment careers in Spain». En: Blossfeld, Hans-Peter y DroBNIC, Sonja. Careers of couples in contemporary society: From male breadwinner to dual earner families. Nueva York: Oxford University Press.

GonZÁlez-López, María José y Jurado, Teresa (2009). “¿Cuándo se implican los hombres en las tareas domésticas? Un análisis de la Encuesta de Empleo del Tiempo». Panorama Social, 65-81.

GrEENSTEIN, Theodore (1996). "Gender ideology and perceptions of the fairness of the division of household labor: Effects on marital quality». Social Forces, 74 (3), 1029-1042. $<$ https://doi.org/10.1093/sf/74.3.1029>.

- (2000). «Economic dependence, gender and the division of labor in the home: A replication and extension». Journal of Marriage and Family, 62 (2), 322-335. <http://dx.doi.org/10.1111/j.1741-3737.2000.00322.x>.

Goldscheider, Frances y Waite, Linda. (1991). New Families, No Families? The Transformation of the American Home. Berkeley, CA: University of California Press.

GUPTA, Sanjiv (2006). «Her money, her time: Women's earnings and their housework hours». Social Science Research, 35 (4), 975-99. $<$ https://doi.org/10.1016/j.ssresearch.2005.07.003>.

- (2007). «Autonomy, dependence or display? The relationship between married women's earnings and housework». Journal of Marriage and Family, 69 (2), 399-417. <https://doi.org/10.1111/j.1741-3737.2007.00373.x>.

GuPTA, Sanjiv y ASH, Michael (2008). "Whose money, whose time? A nonparametric approach to modeling time spent on housework in the United States». Feminist Economics, 14 (1), 93-120. <https://doi.org/10.1080/13545700701716664>.

Hook, Jennifer (2006). "Care in context: Men's unpaid work in 20 countries, 19652003». American Sociological Review, 71, 639-660. <https://doi.org/10.1177/000312240607100406>.

INE-ChILE InstiTUTO NACIONAL DE EsTADÍsTiCAs (2016a). ENUT Encuesta Nacional Sobre Uso del Tiempo. Documento Metodológico. Santiago de Chile: INE

- (2016b). ENUT Encuesta Nacional Sobre Uso del Tiempo. La Dimensión Personal del Tiempo, ENUT 2015. Santiago de Chile: INE.

Institute For Comparative Survey RESEARCh (2017). World value survey. WVS. Viena. <http://www.worldvaluessurvey.org/wvs.jsp>.

Instituto Nacional de Estadística, INE (2011). Encuesta de Empleo del Tiempo 2009-2010. Metodología. Madrid: INE.

InstiTuto NACIONAL DE EsTAdísTICA Y CENSOS, INDEC (2013). Encuesta sobre Trabajo no Remunerado y Uso del Tiempo Informe técnico. Diseño de registro y estructura de la base de microdatos. Buenos Aires: INDEC.

- (2014). Encuesta sobre Trabajo no Remunerado y Uso del Tiempo. Buenos Aires: INDEC.

Juster, Thomas; OnO, Hiromi y STAFFORD, Frank (2003). «An assessment of alternative measures of time use». Sociological Methodology, 33, 19-54. <https://doi.org/10.1111/j.0081-1750.2003.t01-1-00126.x>.

Kan, Man Yee y PUdneY, Stephen (2008). "Measurement error in stylized and diary data on time use». American Sociological Association, 38, 101-132. <https://doi.org/10.1111/j.1467-9531.2008.00197.x>. 
Kan, Man Ye y GershunY, Jonathan (2010). "Gender segregation and bargaining in domestic labour: Evidence from longitudinal time use data» En: CROMPTON, Rosemary; SCOTT, Jacqueline y LYONNETT, Clare (ed.). Gender inequalities in the 21st Century. Aldershot: Edward Elgar.

Kan, Man Ye; Sullivan, Oriel y Gershuny, Jonathan (2011). «Gender convergence in domestic work: discerning the efects of interactional and institutional barriers from large-scale data». Sociology. $<$ https://doi.org/10.1177/0038038510394014>.

KARAMESSINI, Maria y RUBERY, Jill (2014). Women and austerity: The economic crisis and the future for gender equality. Nueva York: Routledge.

KATZ, Richard (1997). «Representational roles». European Journal of Political Research (32), 211-226. <https://doi.org/10.1111/1475-6765.00339>.

Killewald, Alexandra y Gough, Margaret (2010). «Money isn't everything: wives' earnings and housework time». Social Science Research, 39 (6), 987-1003. <https://doi.org/10.1016/j.ssresearch.2010.08.005>.

KitTERØD, Ragni Hege y LyNGSTAD, Torkild Hovde (2005). «Diary versus questionnaire information on time spent on housework. The case of Norway». International Journal of Time Use Research, 2 (1), 13-32. <https://doi.org/10.13085/eijtur.2.1.13-32>.

KnUdSEN, Knu y Wærness, Kari (2008). «National context and spouses' housework in 34 countries». European Sociological Review, 24 (1) 97-113. <https://doi.org/10.1093/esr/jcm037>.

LaChanCE-Grzela, Mylène y Bouchard, Geneviève (2010). "Why do women do the lion's share of housework? A decade of research». Sex Roles (63), 767-780. <https://doi.org/10.1007/s11199-010-9797-z>.

LAMAUTE-BRISSON, Nathalie (2013). «Redistribuir el cuidado: para un nexo de políticas públicas». En: CALDERÓn, C. (coord.). Redistribuir el cuidado. El desafío de las politicas. Santiago de Chile: ONU.

LEWIS, Jane (2010). "Gender and welfare state change». European Societies, 4 (4), 331-357. <https://doi.org/10.1080/1461669022000022324>.

LOMBARDO, Manuela y LEÓN, Margarita (2014). «Políticas de igualdad de género y sociales en España». Investigaciones Feministas, 5, 13-35. $<$ https://doi.org/10.5209/rev_infe.2014.v5.47986>.

Mannino, Cleilia y Deutsch, Francine (2007). "Changing the division of household labor: A negotiated process between partners». Sex Roles, 56 (5-6), 309-324. <https://doi.org/10.1007/s11199-006-9181-1>.

MarCO, Flavia y Rico, María Nieves (2013). «Cuidado y políticas públicas: debates y estado de situación a nivel regional». En: PAUTASSI, Laura y ZiBECCHI, Carla (coord.). Las fronteras del cuidado. Agenda, derechos e infraestructura. Buenos Aires: Biblios.

MARINI, Margaret y SHELTON, Beth A. (1993). "Measuring household work: Recent experience in the United States». Social Science Research, 22, 361-382. <https://doi.org/10.1006/ssre.1993.1018>.

MARTínEZ, Juliana (2005). «Regímenes de bienestar en América Latina: consideraciones generales e itinerarios regionales». Revista Centroamericana de Ciencias Sociales, $2(2), 41-78$.

Midaglia; Carmen y ANTÍA, Florencia (2017). Bienestar y protección social en América Latina. Abordajes conceptuales y metodológicos para el Mercosur. Paraguay: Instituto Social del Mercosur. 
Moreno, Almudena (2007). Modelos familiares y empleo de la mujer en el Estado de bienestar español. Fundación Alternativas.

Moreno, Sara (2009). "Uso del tiempo, desigualdades sociales y ciclo de vida». Política y Sociedad, 46 (3), 191-202.

- (2015). "The gendered division of housework time: Analysis of time use by type and daily frequency of household tasks». Time \& Society (26-1), 3-27. $<$ https://doi.org/10.1177/0961463x15577269>.

PARKMAN, Allen (2004). «Bargaining over housework: The frustrating situation of secondary wage earners». American Journal of Economics and Sociology, 63 (4), 765-94. <https://doi.org/10.1111/j.1536-7150.2004.00316.x>.

Pinto, Katy y Coltrane, Scott (2009). «Divisions of labor in Mexican origin and Anglo families: Structure and culture». Sex Roles, 60 (7-8), 482-495. <https://doi.org/10.1007/s11199-008-9549-5>.

Press, Julie y TOWNSLEY, Eleanor (1998). «Wives' and husbands' housework reporting. Gender, class and social desirability». Gender and Society, 12 (2), 188-218. <https://doi.org/10.1177/089124398012002005>.

SHAHRA, Razavi (ed.) (2012). Seen, heard and counted: rethinking care in a development context. Londres: Wiley Blackwell. <https://doi.org/10.1002/9781118297261>.

SPITZE, Glenna (1988). «Women's employment and family relations: A review». Journal of Marriage and the Family, 50, 595-618. $<$ https://doi.org/10.2307/352633>.

Rico, María Nieves y Robles, Claudia (2016). Políticas de cuidado en América Latina. Forjando la igualdad. Santiago de Chile: CEPAL.

RIzAVI, Sayyid Salman y SofER, Catherine (2010). "Household division of labor: Is there any escape from traditional gender roles?». Documents de travail du Centre d'Economie de la Sorbonne (septiembre de 2010).

RodríGuEZ, Corina (2015). «El trabajo de cuidado no remunerado en Argentina: un análisis desde la evidencia del Módulo de Trabajo no Remunerado». Documentos de trabajo Políticas públicas y derecho al cuidado, 2. ELA - Equipo Latinoamericano de Justicia y Género.

SALAZAR, Octavio (2016). "The fragility of gender equality policies in Spain». Social Sciences, 5 (2), 1-17. <https://doi.org/10.3390/socsci5020017>.

Salvador, Soledad (2007). «Estudio comparativo de la economía del cuidado en Argentina, Brasil, Chile, Colombia, México y Uruguay». Comercio, género y equidad en América Latina: generando conocimiento para la acción política. Buenos Aires: Red Internacional de Género y Comercio.

SAYER, Liana C. (2010). «Trends in housework». En: TrEAS, Judith y DrobNIC, Sonja. Dividing the domestic: Men, women and household work in cross-national perspective (ed.). Stanford: Stanford University Press. <https://doi.org/10.11126/stanford/9780804763578.001.0001>.

SCHUlz, Florian y GRUNOW, Daniela (2011). "Comparing diary and survey estimates on time use». European Sociological Review, 5 (28), 622-632. $<$ https://doi.org/10.1093/esr/jcr030>.

Sevilla-Sanz, Amudena; Giménez-Nadal, José Ignacio y Fernández, Cristina (2010). "Gender roles and the division of unpaid work in Spanish households». Feminist Economics (14), 137-184. <https://doi.org/10.1080/13545701.2010.531197>. 
Treas, Judith y Drobnic, Sonja (eds.) (2010). Dividing the domestic: Men, women and household work in cross-national perspective. Stanford: Stanford University Press. <https://doi.org/10.11126/stanford/9780804763578.001.0001>.

VACA-TRIgo, Iliana (2015). Pobreza y tiempo destinado al trabajo no remunerado: un círculo vicioso. XVI Encuentro Internacional de Estadísticas de Género: Desafíos estadísticos hacia la implementación de la Agenda de Desarrollo Sostenible. México.

Wallace, Ruth (1989). Feminism and theory. Newbury Park: Sage Publications.

YU, Jia y XIE, Yu (2014). "The varying display of 'gender display'». Chinese Sociological Review, 2 (44), 5-30.

<https://doi.org/10.2753/CSA2162-0555440201>. 